DEPT. OF MATH. UNIV. OF OSLO

\title{
A SINGULAR LIMIT PROBLEM FOR CONSERVATION LAWS RELATED TO THE CAMASSA-HOLM SHALLOW WATER EQUATION
}

\author{
GIUSEPPE MARIA COCLITE AND KENNETH HVISTENDAHL KARLSEN
}

\begin{abstract}
We consider a shallow water equation of Camassa-Holm type, containing nonlinear dispersive effects as well as fourth order dissipative effects. We prove that as the diffusion and dispersion parameters tend to zero, with a condition on the relative balance between these two parameters, smooth solutions of the shallow water equation converge to discontinuous solutions of a scalar conservation law. The proof relies on deriving suitable a priori estimates together with an application of the compensated compactness method in the $L^{p}$ setting.
\end{abstract}

\section{INTRODUCTION}

The aim of this paper is to study rigorously the convergence of solutions $u=u_{\varepsilon, \alpha}$, as $\varepsilon, \alpha \rightarrow 0+$, to the following scalar nonlinear partial differential equation

$$
\begin{aligned}
& \partial_{t} u-\alpha \partial_{t x x}^{3} u+\partial_{x} f(u) \\
& =2 \alpha \partial_{x} u \partial_{x x}^{2} u+\alpha u \partial_{x x x}^{3} u+\varepsilon \partial_{x x}^{2} u-\varepsilon \alpha \partial_{x x x x}^{4} u,
\end{aligned}
$$

where $f$ is a smooth, genuinely nonlinear, and at most quadratically growing function.

At least formally, by taking $\varepsilon=0, \alpha=0$ in (1.1) we recover the nonlinear conservation law

$$
\partial_{t} u+\partial_{x} f(u)=0 \text {. }
$$

It is well known that solutions to nonlinear conservation laws in general form discontinuities, and due to this loss of regularity or blow-up it becomes necessary to work with distributional (weak) solutions. However, since various physical mechanisms have been neglected, weak solutions are not unique, so that an additional condition is needed to single out a unique weak solution. This additional condition is called the entropy condition. The prototype example of a conservation law for which all these features are present is the Burgers equation $\left(f(u)=\frac{u^{2}}{2}\right)$. For an introduction to conservation laws, see for example [21].

In this paper we prove rigorously that $u_{\varepsilon, \alpha}$ converges strongly to a limit function $u$ that is a weak solution $u$ of (1.2), as long as the parameters $\alpha$ and $\varepsilon$ are kept in balance in the sense that

$$
\alpha=O\left(\varepsilon^{4}\right) .
$$

The convergence takes place in $L_{\text {loc }}^{p}$, for each $2<p<4$. Under the stronger condition

$$
\alpha=o\left(\varepsilon^{4}\right),
$$

we prove that the limit $u$ dissipates energy, that is, the entropy inequality

$$
\partial_{t}\left(\frac{u^{2}}{2}\right)+\partial_{x} q(u) \leq 0, \quad q^{\prime}(u)=u f^{\prime}(u),
$$

holds in the weak sense. When $f$ is globally Lipschitz continuous we prove similar results with $L^{4}$ replaced by $L^{2}$ and $\alpha=O\left(\varepsilon^{4}\right), o\left(\varepsilon^{4}\right)$ replaced by $\alpha=O\left(\varepsilon^{2}\right), o\left(\varepsilon^{2}\right)$ respectively.

Date: April 18, 2005.

2000 Mathematics Subject Classification. 35G25, 35L65, 35B25.

Key words and phrases. shallow water equation, Burgers equation, conservation law, entropy condition, singular limit, compensated compactness.

The research of K. H. Karlsen is supported by the Research Council of Norway through the BeMatA program and an Outstanding Young Investigators Award and by the European network HYKE, contract HPRN-CT-2002-00282. 
Before we discuss the motivation for studying the singular limit problem associated with (1.1), we give a brief overview of previous work on singular perturbation problems for conservation laws.

The classical problem is that of vanishing viscosity $\varepsilon \rightarrow 0+$ in solutions $u=u_{\varepsilon}$ to the uniformly parabolic equations

$$
\partial_{t} u+\partial_{x} f(u)=\varepsilon \partial_{x x}^{2} u .
$$

While a solution to the conservation law possesses discontinuities, the solution to the above parabolic equation is always smooth thanks to the regularization effect of the operator $\varepsilon \partial_{x x}^{2} u$. The vanishing viscosity limit lies at the heart of the matter of the famous theory of Kružkov [27]. This theory says, among many other things, that $u_{\varepsilon}$ converges strongly (in $L_{\mathrm{loc}}^{p}, 1 \leq p<\infty$ ) to a limit $u$, which is a weak solution of the conservation law and moreover satisfies an infinite family of entropy inequalities of the form

$$
\partial_{t}|u-c|+\partial_{x}[\operatorname{sign}(u-c)(f(u)-f(c))] \leq 0, \quad \forall c \in \mathbb{R},
$$

which is the so-called Kružkov's entropy condition. The key ingredients in this theory are the maximum principle and $B V$ estimates, which can be viewed as consequences of the fundamental $L^{1}$ contraction property shared by entropy solutions.

A different singular perturbation problem is that of vanishing dispersion $\delta \rightarrow 0+$ in solutions $u=u_{\delta}$ to the generalized $\mathrm{KdV}$ equation

$$
\partial_{t} u+\partial_{x} f(u)=-\delta \partial_{x x x}^{3} u
$$

It is known that when $t$ exceeds the time of shock formation for the conservation law (1.2), the dispersive solution $u=u_{\delta}$ behaves in an oscillatory manner. Moreover, as $\delta \rightarrow 0+$ the amplitude of these oscillations stays bounded but does not tend to zero, and its wave length is of order $\delta$. Hence, $u_{\delta}$ converges only weakly to some limit function $u$ as $\delta \rightarrow 0+$, which does not satisfy the conservation law (1.2). We refer to Lax and Levermore [28, 29, 30] for more information on the $\mathrm{KdV}$ small dispersion limit problem.

Schonbeck [39] studied the combined singular limit problem of vanishing diffusion $\varepsilon \rightarrow 0+$ and vanishing dispersion $\delta \rightarrow 0+$ (with $\varepsilon$ and $\delta$ kept in balance) in solutions $u=u_{\varepsilon, \delta}$ to

$$
\begin{aligned}
& \partial_{t} u+\partial_{x} f(u)=\varepsilon \partial_{x x}^{2} u-\delta \partial_{x x x}^{3} u, \\
& \partial_{t} u-\delta \partial_{t x x}^{3} u+\partial_{x} f(u)=\varepsilon \partial_{x x}^{2} u .
\end{aligned}
$$

The first equation in (1.3) is often referred to as the generalized KdV-Burgers equation, while the second equation is often referred to as the generalized BBM-Burgers equation (see for [2] for the BBM equation). For these equations $L^{1}$ contraction principles, maximum principles, or $B V$ estimates are not available. Instead Schonbek [39] developed and used the $L^{p}$ version of the compensated compactness method [42]. Schonbek's convergence results for the (generalized) KdVBurgers equation have been extended in various directions by LeFloch and Natalini [32], Correia and Lefloch [14], Kondo and Lefloch [26], and also Hwang and Tzavaras [24].

Depending on the relationship between $\varepsilon$ and $\delta$ when performing the limit $\varepsilon \rightarrow 0+, \delta \rightarrow 0+$, different results are obtained. For example, for the generalized KdV-Burgers equation with a globally Lipschitz flux $f$, the following results are known [26]: $(i) \delta \ll \varepsilon^{2}$. The solutions $u_{\varepsilon, \delta}$ then converge strongly to a Kružkov entropy solution of (1.2). (ii) $\delta \gg \varepsilon^{2}$. The solutions $u_{\varepsilon, \delta}$ then become highly oscillatory and do not converge strongly to a solution of (1.2) (cf. also the discussion above on the $\mathrm{KdV}$ type equation). (iii) $\delta=C \varepsilon^{2}$ with $C$ fixed. In this intermediate regime, the solutions $u_{\varepsilon, \delta}$ converge strongly (in $L_{\mathrm{loc}}^{p}, 1<p<2$ ) but in general not to a Kružkov entropy solution of (1.2), unless $f$ is convex.

In the intermediate region it is possible that the limit function contains so-called non-classical shock waves that dissipate the single entropy $\eta(u)=\frac{1}{2} u^{2}$ (the "energy") but not all convex entropies. Hence non-classical shock waves may violate the entropy condition of Oleĭnik and in that sense they are undercompressive. We refer to the book of LeFloch [31] for an introduction to the highly active field of non-classical shocks in (systems of) conservation laws.

Lucier [35] proved that the solutions $u=u_{\varepsilon, \delta}$ to the equation

$$
\partial_{t} u-\delta \partial_{t x x}^{3} u+\partial_{x} f(u)=\varepsilon \partial_{x x}^{2} g(u)
$$


converge strongly (in $L_{\mathrm{loc}}^{p}, 1 \leq p<\infty$ ) to a Kružkov entropy solution of (1.2) provided that the ratio $\alpha=\varepsilon^{2} / \delta$ is kept fixed as $\varepsilon, \delta \rightarrow 0$, and the functions $f$ and $g$ are globally Lipschitz continuous and $\varepsilon g(u) \pm \sqrt{\delta} f(u)$ are nondecreasing in $u$. Observe that if $f, g$ are continuously differentiable, this latter condition is equivalent to the relation $\varepsilon g^{\prime}(u) \geq \sqrt{\delta}\left|f^{\prime}(u)\right|$ for all $u$. The above relation thus says that diffusion effects must dominate the interaction between nonlinear convection and dispersion terms. Under these circumstances, Lucier was able to use the theory of $L^{1}$ contractive semigroups, the maximum principle, and $B V$ estimates to prove his results. His analysis should be compared with the $L^{p}$ compensated compactness analysis for the (generalized) BBM-Burgers equation by Schonbeck [39], who proved strong convergence to a weak solution under the assumption that $\delta=O\left(\varepsilon^{4}\right)$.

To conclude this (incomplete) overview of singular limit problems for conservation laws, we mention that there are also some papers that study the singular limit $\varepsilon \rightarrow 0+, \delta \rightarrow 0+$ for solutions $u=u_{\varepsilon, \delta}$ to fourth (or higher) order equations of the form

$$
\partial_{t} u+\partial_{x} f(u)=\varepsilon \partial_{x x}^{2} u-\delta \partial_{x x x x}^{4} u,
$$

see Cassis [4], Zhao [45], Hwang [23], and also Tadmor [41].

Before returning to (1.1), let us recall that the $\mathrm{KdV}$ (-Burgers) and BBM(-Burgers) equations are used as models for the study of weakly nonlinear unidirectional long waves. They arise in physical contexts in which there is a balance between nonlinearity and dispersion at leading order. For example, they model surface waves of small amplitude and long wavelength on shallow water. The $\mathrm{KdV}$ and BBM equations are asymptotically equivalent to the neglected order of approximation. However, while the $\mathrm{KdV}$ equation is completely integrable and possesses solitary wave solutions that are solitons, the BBM equation does not share these properties. On the other hand, for the $\mathrm{KdV}$ equation the linearized dispersion relation is not bounded for large wave numbers, whereas it is for the BBM equation (in fact it tends to zero as the wave number tends to infinity).

In the early 1990 s a new equation for shallow water waves entered the arena, namely the socalled Camassa-Holm equation [3]. This nonlinearly dispersive wave equation, which has received a considerable amount of attention in recent years, takes the form

$$
\partial_{t} u-\alpha \partial_{t x x}^{3} u+\kappa u \partial_{x} u+3 u \partial_{x} u=2 \alpha \partial_{x} u \partial_{x x}^{2} u+\alpha u \partial_{x x x}^{3} u .
$$

Observe that (1.5) can be obtained by taking $\varepsilon=0$ and $f(u)=\kappa u+\frac{3}{2} u^{2}$ in (1.1). As already alluded to, in one interpretation, the Camassa-Holm equation models the propagation of unidirectional shallow water waves on a flat bottom, and then $u(t, x)$ represents the fluid velocity at time $t$ in the horizontal direction $x[3,25]$. Within this context, $\alpha>0$ is a length scale (related to the shallowness) and $\kappa \geq 0$ is a constant that is proportional to the square root of water depth. In another interpretation, Dai [15] derived (1.5) (in a more general form) as an equation describing finite length, small amplitude radial deformation waves in cylindrical compressible hyperelastic rods. For a discussion of Camassa-Holm and related equations as well as their analytical/numerical properties, see the recent paper by Holm and Staley [22].

The Camassa-Holm equation goes beyond the linear order $\mathrm{KdV}$ and BBM equations in the sense that (1.5) appears as a water wave equation at quadratic order in an asymptotic expansion for unidirectional shallow water waves modeled by the incompressible Euler equations, whereas the KdV and BBM equations appear at first order in this asymptotic expansion [3, 25]. Indeed, (1.5) takes into account nonlinear dispersive effects in addition to the terms associated with the BBM equation, and for that reason it can be viewed as a generalization of the BBM equation. The Camassa-Holm equation (1.5) contains higher order nonlinear/nonlocal balances not present in the $\mathrm{KdV}$ and BBM equations. Moreover, as in the BBM equation but not in the KdV equation, the linear dispersion relation in the Camassa-Holm equation remains bounded for large wave numbers.

The Camassa-Holm equation possesses many astonishing properties. Let us mention its biHamiltonian structure (and thus an infinite number of conservation laws) [19, 3] and that, as is the case with the $\mathrm{KdV}$ equation but not the BBM equation, it is completely integrable $[3,1,12,8]$. Moreover, when $\kappa=0$ it has an infinite number of non-smooth solitary wave solutions called peakons (due to the discontinuity of their first derivatives at the wave peak), which interact like solitons. Although the $\mathrm{KdV}$ equation admits solitary waves that are solitons, it does not model 
wave breaking. The Camassa-Holm equation is remarkable in the sense that it admits soliton solutions and at the same time allow for wave breaking.

From a mathematical point of view the Camassa-Holm equation is by now well studied. Local well-posedness results are proved in $[9,20,33,38]$. It is also known that there exist global solutions for a certain class of initial data and also solutions that blow up in finite time for a large class of initial data $[7,9,11]$. Existence and uniqueness results for global weak solutions of (1.5) have been proved by Constantin and Escher [10], Constantin and Molinet [13], and Xin and Zhang [43, 44], see also Danchin $[16,17]$. The generalized Camassa-Holm equation (1.1) with $\varepsilon=0$ was analyzed recently in [5], while the easier case when $\varepsilon>0$ (so that smooth solutions exist) is contained as a special case of a more general class of equations analyzed in [6].

We recall that weak solutions of, say, (1.5) are sought in the class $L^{\infty}\left(\mathbb{R}_{+} ; H^{1}(\mathbb{R})\right)$ and is based on a distributional interpretation of the system

$$
\partial_{t} u+u \partial_{x} u+\partial_{x} P=0, \quad-\alpha \partial_{x x}^{2} P+P=u^{2}+\frac{\alpha}{2}\left(\partial_{x} u\right)^{2},
$$

where it may be instructive to recall that the Green's function of the elliptic operator $P \mapsto$ $-\alpha \partial_{x x}^{2} P+P$ is $\frac{e^{-|x| / \sqrt{\alpha}}}{2 \sqrt{\alpha}}$. It is not difficult to see that the scalar equation (1.5) is equivalent to the system (1.6), at least when the solutions are smooth.

One way to prove existence of a weak solution to (1.6) goes via the vanishing viscosity method, that is, one attempts to take the limit $\varepsilon \rightarrow 0+$ in smooth solutions $u=u_{\varepsilon}$ to the system

$$
\partial_{t} u+u \partial_{x} u+\partial_{x} P=\varepsilon \partial_{x x}^{2} u, \quad-\alpha \partial_{x x}^{2} P+P=u^{2}+\frac{\alpha}{2}\left(\partial_{x} u\right)^{2} .
$$

We refer to [43] for an implementation of this strategy, which is highly nontrivial due to the nonlinear nature of the system (1.7). Writing out (1.7) as a scalar equation yields the fourth order equation (1.1) with $f(u)=\frac{3 u^{2}}{2}$. In this paper we will consider equation (1.1) with a "general" $f$, which can be viewed as a generalized Camassa-Holm equation that accounts for nonlinear dispersive effects as well as (linear) dissipative effects.

In passing, let us mention another important ingredient in the mathematical treatment of the Camassa-Holm equation, namely the "vorticity caricature" $m:=u-\alpha \partial_{x x}^{2} u$ (see, e.g., [10, 13]), which satisfies the equation

$$
\partial_{t} m+u \partial_{x} m+2 m \partial_{x} u=0 .
$$

Assuming that $\left.m\right|_{t=0}$ is a bounded nonnegative measure, it is not hard to see that the solution $m(t, \cdot)$ to $(1.8)$ remains a nonnegative measure at later times. Consequently, one can easily bound $\partial u / \partial x$ in $L^{\infty}$ and $\partial^{2} u / \partial x^{2}$ in $L^{1}$. These rather strong a priori bounds can then be used as basis for proving existence (and uniqueness) of an energy conservative weak solution [10, 13].

Unfortunately, in the present paper we can neither exploit the "system formulation" nor the "vorticity formulation" of the Camassa-Holm equation. Indeed, most of the a priori estimates and technical tools associated with these formulations are too strong to capture the (discontinuous) limit of solutions $u_{\varepsilon, \alpha}$ to (1.1) as the diffusion and dispersion parameters tend to zero. Instead, we will derive suitable a priori estimates by working directly on the scalar equation (1.1). Following Schonbek [39], we then use the $L^{p}$ compensated compactness method to obtain strong convergence of a subsequence of $\left\{u_{\varepsilon, \alpha}\right\}_{\varepsilon, \alpha}$ to a limit function that is a weak solution of the conservation law (1.2). Here we reiterate that due to the (nonlinear) dispersion terms we do not have uniform $L^{1}, L^{\infty}, B V$ estimates at our disposal. Depending on the relationship between $\alpha$ and $\beta$, we also prove that the limit dissipates the entropy $\eta(u)=\frac{u^{2}}{2}$. However, different from KdV-Burgers type singular limit problems, it seems that the limit function $u$ does not dissipate all convex entropies, even if we impose a stronger condition on the balance between $\varepsilon$ and $\alpha$.

The remaining part of this paper is organized as follows: In Section 2 we give precise conditions on the data our problems and state our main theorem (Theorem 2.1). Some tools needed for the convergence analysis are recalled in Section 3. A priori estimates are derived in Section 4, while the proof of Theorem 2.1 is given in Section 5 . 


\section{Statement of THE MAIN RESUlt}

Consider the nonlinear conservation law

$$
\partial_{t} u+\partial_{x} f(u)=0, \quad(t, x) \in \mathbb{R}_{+} \times \mathbb{R},
$$

which is supplemented with initial data

$$
u(0, x)=u_{0}(x), \quad x \in \mathbb{R} .
$$

Consider the Camassa-Holm type equation

$$
\begin{aligned}
& \partial_{t} u_{\varepsilon, \alpha}-\alpha \partial_{t x x}^{3} u_{\varepsilon, \alpha}+\partial_{x} f\left(u_{\varepsilon, \alpha}\right) \\
& =2 \alpha \partial_{x} u_{\varepsilon, \alpha} \partial_{x x}^{2} u_{\varepsilon, \alpha}+\alpha u_{\varepsilon, \alpha} \partial_{x x x}^{3} u_{\varepsilon, \alpha}+\varepsilon \partial_{x x}^{2} u_{\varepsilon, \alpha}-\varepsilon \alpha \partial_{x x x x}^{4} u_{\varepsilon, \alpha}, \quad(t, x) \in \mathbb{R}_{+} \times \mathbb{R}
\end{aligned}
$$

which is supplemented with initial data

$$
u_{\varepsilon, \alpha}(0, x)=u_{\varepsilon, \alpha, 0}(x), \quad x \in \mathbb{R} .
$$

We assume that the flux $f: \mathbb{R} \rightarrow \mathbb{R}$ is a $C^{2}$ function satisfying

$$
\left|f^{\prime}(u)\right| \leq \kappa_{0}|u|, \quad|f(u)| \leq \kappa_{1}|u|^{2}, \quad u \in \mathbb{R},
$$

or

$$
\left|f^{\prime}(u)\right| \leq \kappa_{2}, \quad|f(u)| \leq \kappa_{3}|u|, \quad u \in \mathbb{R}
$$

for some constants $\kappa_{0}, \kappa_{1}, \kappa_{2}, \kappa_{3}>0$, and the genuinely nonlinearity condition

$$
\text { meas }\left\{u \in \mathbb{R}: f^{\prime \prime}(u)=0\right\}=0 .
$$

We assume that the initial function $u_{0}$ satisfies

$$
\begin{aligned}
& u_{0} \in L^{4}(\mathbb{R}) \cap L^{2}(\mathbb{R}), \text { if }(2.5) \text { holds, } \\
& u_{0} \in L^{2}(\mathbb{R}), \text { if }(2.6) \text { holds. }
\end{aligned}
$$

We assume that the initial functions $u_{\varepsilon, \alpha, 0}$ satisfy

$$
\begin{aligned}
& u_{\varepsilon, \alpha, 0} \in H^{2}(\mathbb{R}), u_{\varepsilon, \alpha, 0} \rightarrow u_{0} \text { in } L^{2}(\mathbb{R}) \cap L^{4}(\mathbb{R}) \text { as } \varepsilon, \alpha \rightarrow 0, \text { if }(2.5) \text { holds, } \\
& u_{\varepsilon, \alpha, 0} \in H^{2}(\mathbb{R}), u_{\varepsilon, \alpha, 0} \rightarrow u_{0} \text { in } L^{2}(\mathbb{R}) \text { as } \varepsilon, \alpha \rightarrow 0, \text { if }(2.6) \text { holds }
\end{aligned}
$$

and

$$
\begin{aligned}
& \left\|u_{\varepsilon, \alpha, 0}\right\|_{L^{1}(\mathbb{R})}+\left\|u_{\varepsilon, \alpha, 0}\right\|_{L^{2}(\mathbb{R})}+\left\|u_{\varepsilon, \alpha, 0}\right\|_{L^{4}(\mathbb{R})}+\left(\alpha+\sqrt{\alpha+\varepsilon^{2}}\right)\left\|\partial_{x} u_{\varepsilon, \alpha, 0}\right\|_{L^{2}(\mathbb{R})} \\
& +\sqrt{\alpha^{2}+\varepsilon^{2} \alpha}\left\|\partial_{x x}^{2} u_{\varepsilon, \alpha, 0}\right\|_{L^{2}(\mathbb{R})} \leq C_{0}, \quad \varepsilon, \alpha>0, \quad \text { if }(2.5) \text { holds, } \\
& \left\|u_{\varepsilon, \alpha, 0}\right\|_{L^{1}(\mathbb{R})}+\left\|u_{\varepsilon, \alpha, 0}\right\|_{L^{2}(\mathbb{R})}+\left(\alpha+\sqrt{\alpha+\varepsilon^{2}}\right)\left\|\partial_{x} u_{\varepsilon, \alpha, 0}\right\|_{L^{2}(\mathbb{R})} \\
& +\sqrt{\alpha^{2}+\varepsilon^{2} \alpha}\left\|\partial_{x x}^{2} u_{\varepsilon, \alpha, 0}\right\|_{L^{2}(\mathbb{R})} \leq C_{0}, \quad \varepsilon, \alpha>0, \quad \text { if }(2.6) \text { holds, }
\end{aligned}
$$

for some constant $C_{0}>0$ that is independent of $\varepsilon, \alpha$.

The main result of this paper is the following theorem:

Theorem 2.1. Assume that (2.7)-(2.10) hold.

If (2.5) is satisfied and

$$
\alpha=O\left(\varepsilon^{4}\right),
$$

then there exist two sequences $\left\{\varepsilon_{k}\right\}_{k \in \mathbb{N}},\left\{\alpha_{k}\right\}_{k \in \mathbb{N}}$, with $\varepsilon_{k}, \alpha_{k} \rightarrow 0$, and a limit function

$$
u \in L^{\infty}\left(\mathbb{R}_{+} ; L^{4}(\mathbb{R}) \cap L^{2}(\mathbb{R})\right),
$$

such that

(i) $u_{\varepsilon_{k}, \alpha_{k}} \rightarrow u$ strongly in $L_{\mathrm{loc}}^{p}\left(\mathbb{R}_{+} \times \mathbb{R}\right)$, for each $1 \leq p<4$, and

(ii) $u$ is a distributional solution of the Cauchy problem (2.1), (2.2). 
If (2.6) is satisfied and

$$
\alpha=O\left(\varepsilon^{2}\right)
$$

then

(ii) holds, and

$$
u \in L^{\infty}\left(\mathbb{R}_{+} ; L^{2}(\mathbb{R})\right)
$$

(iii) $u_{\varepsilon_{k}, \alpha_{k}} \rightarrow u$ strongly in $L_{\text {loc }}^{p}\left(\mathbb{R}_{+} \times \mathbb{R}\right)$, for each $1 \leq p<2$.

Moreover, if (2.5) holds and

$$
\alpha=o\left(\varepsilon^{4}\right),
$$

or if (2.6) holds and

$$
\alpha=o\left(\varepsilon^{2}\right),
$$

we have

(iv) dissipation of the energy, that is,

$$
\partial_{t}\left(\frac{u^{2}}{2}\right)+\partial_{x} q(u) \leq 0 \text { in the weak sense on } \mathbb{R}_{+} \times \mathbb{R},
$$

where $q: \mathbb{R} \rightarrow \mathbb{R}$ is defined by $q^{\prime}(u)=u f^{\prime}(u)$,

Remark 2.1. We proved in [6] the existence of a unique smooth solution $u_{\varepsilon, \alpha}$ to (2.3), (2.4), which moreover depends continuously on the initial condition $u_{\varepsilon, \alpha, 0}$ and on the function $f$.

Remark 2.2. Different from KdV-Burgers type singular limit problems [32, 14, 26, 24, 23], we are not able to prove that the limit function constructed in Lemma 5.2 dissipates all convex entropies, even if we were to impose a stronger condition on the balance between $\varepsilon$ and $\alpha$ than we did in Theorem 2.1. Morever, we have not be able to derive any uniform $L^{1}$ bound on $u_{\varepsilon, \alpha}$.

In the convex case $\left(f(u)=\frac{u^{2}}{2}\right)$ a recent result of De Lellis, Otto, and Westdickenberg [18] (see also an earlier result by Panov [37] in the $L^{\infty}$ context) shows that the satisfaction of the entropy inequality for a single strictly convex entropy is enough to characterize an $L^{4}$ Kružkov entropy solution to Burgers' equation. We refer to Szepessy [40] for the $L^{1} \cap L^{p}$ version of Kružkov's well-posedness theory [27].

\section{Mathematical PReliminaries}

Later we will use Schonbek's $L^{p}$ version [39] of the compensated compactness method [42]. However, to avoid assuming strict convexity of the flux function, we will use a refinement of Schonbek's method found in [34], which is stated next.

Lemma 3.1. Let $\Omega$ be a bounded open subset of $\mathbb{R}_{+} \times \mathbb{R}$. Let $f \in C^{2}(\mathbb{R})$ satisfy

$$
|f(u)| \leq C|u|^{s+1} \text { for } u \in \mathbb{R}, \quad\left|f^{\prime}(u)\right| \leq C|u|^{s} \text { for } u \in \mathbb{R},
$$

for some $s \geq 0$, and

$$
\text { meas }\left\{u \in \mathbb{R}: f^{\prime \prime}(u)=0\right\}=0 .
$$

Define functions $I_{l}, f_{l}, F_{l}: \mathbb{R} \rightarrow \mathbb{R}$ as follows:

$$
\left\{\begin{array}{l}
I_{l} \in C^{2}(\mathbb{R}),\left|I_{l}(u)\right| \leq|u| \text { for } u \in \mathbb{R},\left|I_{l}^{\prime}(u)\right| \leq 2 \text { for } u \in \mathbb{R} \\
\left|I_{l}(u)\right| \leq|u| \text { for }|u| \leq l, I_{l}(u)=0 \text { for }|u| \geq 2 l
\end{array}\right.
$$

and

$$
f_{l}(u)=\int_{0}^{u} I_{l}^{\prime}(\zeta) f^{\prime}(\zeta) d \zeta, \quad F_{l}(u)=\int_{0}^{u} f_{l}^{\prime}(\zeta) f^{\prime}(\zeta) d \zeta .
$$

Suppose $\left\{u_{n}\right\}_{n=1}^{\infty} \subset L^{2(s+1)}(\Omega)$ is such that the two sequences

$$
\left\{\partial_{t} I_{l}\left(u_{n}\right)+\partial_{x} f_{l}\left(u_{n}\right)_{x}\right\}_{n=1}^{\infty}, \quad\left\{\partial_{t} f_{l}\left(u_{n}\right)+\partial_{x} F_{l}\left(u_{n}\right)\right\}_{n=1}^{\infty}
$$

of distributions belong to a compact subset of $H_{\mathrm{loc}}^{-1}(\Omega)$, for each fixed $l>0$. 
Then there exists a subsequence of $\left\{u_{n}\right\}_{n=1}^{\infty}$ that converges to a function $u \in L^{2(s+1)}(\Omega)$ strongly in $L^{r}(\Omega)$ for any $1 \leq r<2(s+1)$.

When we later apply Lemma 3.1, the following lemma of Murat [36]) is useful:

Lemma 3.2. Let $\Omega$ be a bounded open subset of $\mathbb{R}^{N}, N \geq 2$. Suppose the sequence $\left\{\mathcal{L}_{n}\right\}_{n=1}^{\infty}$ of distributions is bounded in $W^{-1, \infty}(\Omega)$. Suppose also that

$$
\mathcal{L}_{n}=\mathcal{L}_{n}^{1}+\mathcal{L}_{n}^{2}
$$

where $\left\{\mathcal{L}_{n}^{1}\right\}_{n=1}^{\infty}$ lies in a compact subset of $H_{\mathrm{loc}}^{-1}(\Omega)$ and $\left\{\mathcal{L}_{n}^{2}\right\}_{n=1}^{\infty}$ lies in a bounded subset of $\mathcal{M}_{\mathrm{loc}}(\Omega)$. Then $\left\{\mathcal{L}_{n}\right\}_{n=1}^{\infty}$ lies in a compact subset of $H_{\mathrm{loc}}^{-1}(\Omega)$.

\section{A PRIORI EStimates}

The main results in this section are collected in two lemmas. The first one contains the energy estimate that yields $L^{2}$ estimates for various quantities involving partial derivatives up to order two, while the second one contains additional $L^{4} / L^{2}$ bounds on various quantities containing partial derivatives of $u_{\varepsilon, \alpha}$ up to order three.

Lemma 4.1. Assume that (2.9) and (2.10) hold. Then the following identity holds

$$
\begin{aligned}
& \int_{\mathbb{R}}\left[\left(u_{\varepsilon, \alpha}(t, x)\right)^{2}+\alpha\left(\partial_{x} u_{\varepsilon, \alpha}(t, x)\right)^{2}\right] d x \\
& \quad+2 \varepsilon \int_{0}^{t} \int_{\mathbb{R}}\left[\left(\partial_{x} u_{\varepsilon, \alpha}(\tau, x)\right)^{2}+\alpha\left(\partial_{x x}^{2} u_{\varepsilon, \alpha}(\tau, x)\right)^{2}\right] d x d \tau \\
& =\int_{\mathbb{R}}\left[u_{0, \varepsilon, \alpha}(x)^{2}+\alpha\left(\partial_{x} u_{0, \varepsilon, \alpha}(x)\right)^{2}\right] d x,
\end{aligned}
$$

for each $t \geq 0, \varepsilon, \alpha>0$. In particular,

$$
\begin{aligned}
& \left\|u_{\varepsilon, \alpha}\right\|_{L^{\infty}\left(\mathbb{R}_{+} ; L^{2}(\mathbb{R})\right)}, \sqrt{\alpha}\left\|\partial_{x} u_{\varepsilon, \alpha}\right\|_{L^{\infty}\left(\mathbb{R}_{+} ; L^{2}(\mathbb{R})\right)} \leq C_{0} \\
& \sqrt{\varepsilon}\left\|\partial_{x} u_{\varepsilon, \alpha}\right\|_{L^{2}\left(\mathbb{R}_{+} \times \mathbb{R}\right)}, \sqrt{\varepsilon \alpha}\left\|\partial_{x x}^{2} u_{\varepsilon, \alpha}\right\|_{L^{2}\left(\mathbb{R}_{+} \times \mathbb{R}\right)} \leq \frac{C_{0}}{\sqrt{2}} .
\end{aligned}
$$

Moreover,

$$
\left\|u_{\varepsilon, \alpha}\right\|_{L^{\infty}\left(\mathbb{R}_{+} \times \mathbb{R}\right)} \leq \sqrt{2} C_{0} \alpha^{-1 / 4}
$$

Proof. Multiplying (2.3) by $u_{\varepsilon, \alpha}$ and integrating on $\mathbb{R}$ we have

$$
\begin{aligned}
& \int_{\mathbb{R}} u_{\varepsilon, \alpha} \partial_{t} u_{\varepsilon, \alpha} d x-\alpha \int_{\mathbb{R}} u_{\varepsilon, \alpha} \partial_{t x x}^{3} u_{\varepsilon, \alpha} d x+\int_{\mathbb{R}} f^{\prime}\left(u_{\varepsilon, \alpha}\right) u_{\varepsilon, \alpha} \partial_{x} u_{\varepsilon, \alpha} d x \\
& =2 \alpha \int_{\mathbb{R}} u_{\varepsilon, \alpha} \partial_{x} u_{\varepsilon, \alpha} \partial_{x x}^{2} u_{\varepsilon, \alpha} d x+\alpha \int_{\mathbb{R}} u_{\varepsilon, \alpha}^{2} \partial_{x x x}^{3} u_{\varepsilon, \alpha} d x \\
& \quad+\varepsilon \int_{\mathbb{R}} u_{\varepsilon, \alpha} \partial_{x x}^{2} u_{\varepsilon, \alpha} d x-\varepsilon \alpha \int_{\mathbb{R}} u_{\varepsilon, \alpha} \partial_{x x x x}^{4} u_{\varepsilon, \alpha} d x .
\end{aligned}
$$

Observe that

$$
\begin{gathered}
\int_{\mathbb{R}} u_{\varepsilon, \alpha} \partial_{t} u_{\varepsilon, \alpha} d x-\alpha \int_{\mathbb{R}} u_{\varepsilon, \alpha} \partial_{t x x}^{3} u_{\varepsilon, \alpha} d x=\frac{d}{d t} \int_{\mathbb{R}} \frac{u_{\varepsilon, \alpha}^{2}+\alpha\left(\partial_{x} u_{\varepsilon, \alpha}\right)^{2}}{2} d x, \\
\varepsilon \int_{\mathbb{R}} u_{\varepsilon, \alpha} \partial_{x x}^{2} u_{\varepsilon, \alpha} d x-\varepsilon \alpha \int_{\mathbb{R}} u_{\varepsilon, \alpha} \partial_{x x x x}^{4} u_{\varepsilon, \alpha} d x=-\varepsilon \int_{\mathbb{R}}\left(\left(\partial_{x} u_{\varepsilon, \alpha}\right)^{2}+\alpha\left(\partial_{x x}^{2} u_{\varepsilon, \alpha}\right)^{2}\right) d x, \\
2 \alpha \int_{\mathbb{R}} u_{\varepsilon, \alpha} \partial_{x} u_{\varepsilon, \alpha} \partial_{x x}^{2} u_{\varepsilon, \alpha} d x+\alpha \int_{\mathbb{R}} u_{\varepsilon, \alpha}^{2} \partial_{x x x}^{3} u_{\varepsilon, \alpha} d x-\int_{\mathbb{R}} f^{\prime}\left(u_{\varepsilon, \alpha}\right) u_{\varepsilon, \alpha} \partial_{x} u_{\varepsilon, \alpha} d x=0 .
\end{gathered}
$$

Hence, we can rewrite (4.5) in the following way

$$
\frac{d}{d t} \int_{\mathbb{R}}\left(u_{\varepsilon, \alpha}^{2}+\alpha\left(\partial_{x} u_{\varepsilon, \alpha}\right)^{2}\right) d x+2 \varepsilon \int_{\mathbb{R}}\left(\left(\partial_{x} u_{\varepsilon, \alpha}\right)^{2}+\alpha\left(\partial_{x x}^{2} u_{\varepsilon, \alpha}\right)^{2}\right) d x=0
$$

Integrating (4.6) on the interval $[0, t]$, we get (4.1). 
To prove (4.4), we use (4.2) and the Hölder inequality as follows:

$$
\begin{aligned}
u_{\varepsilon, \alpha}^{2}(t, x) & \leq 2 \int_{\mathbb{R}}\left|u_{\varepsilon, \alpha} \partial_{x} u_{\varepsilon, \alpha}\right| d x \\
& \leq 2\left\|u_{\varepsilon, \alpha}(t, \cdot)\right\|_{L^{2}(\mathbb{R})}\left\|\partial_{x} u_{\varepsilon, \alpha}(t, \cdot)\right\|_{L^{2}(\mathbb{R})} \\
& \leq \frac{2 C_{0}^{2}}{\sqrt{\alpha}}, \quad \text { for every } t \geq 0, x \in \mathbb{R}, \varepsilon, \alpha>0 .
\end{aligned}
$$

Lemma 4.2. Assume that (2.9) and (2.10) hold.

If (2.5) and (2.11) are satisfied, then

i) the family $\left\{u_{\varepsilon, \alpha}\right\}_{\varepsilon, \alpha}$ is bounded in $L^{\infty}\left(\mathbb{R}_{+} ; L^{4}(\mathbb{R})\right)$;

ii) the following families are bounded in $L^{\infty}\left(\mathbb{R}_{+} ; L^{2}(\mathbb{R})\right)$ :

$$
\begin{aligned}
& \left\{\sqrt{\alpha} \partial_{x} u_{\varepsilon, \alpha}\right\}_{\varepsilon, \alpha}, \quad\left\{\varepsilon \partial_{x} u_{\varepsilon, \alpha}\right\}_{\varepsilon, \alpha}, \\
& \left\{\alpha \partial_{x x}^{2} u_{\varepsilon, \alpha}\right\}_{\varepsilon, \alpha}, \quad\left\{\varepsilon \sqrt{\alpha} \partial_{x x}^{2} u_{\varepsilon, \alpha}\right\}_{\varepsilon, \alpha} ;
\end{aligned}
$$

iii) the following families are bounded in $L^{2}\left(\mathbb{R}_{+} \times \mathbb{R}\right)$ :

$$
\begin{aligned}
& \left\{\sqrt{\varepsilon} \partial_{t} u_{\varepsilon, \alpha}\right\}_{\varepsilon, \alpha}, \quad\left\{\sqrt{\varepsilon \alpha} \partial_{t x}^{2} u_{\varepsilon, \alpha}\right\}_{\varepsilon, \alpha}, \quad\left\{\sqrt{\varepsilon} u_{\varepsilon, \alpha} \partial_{x} u_{\varepsilon, \alpha}\right\}_{\varepsilon, \alpha} \\
& \left\{\sqrt{\varepsilon \alpha} u_{\varepsilon, \alpha} \partial_{x x}^{2} u_{\varepsilon, \alpha}\right\}_{\varepsilon, \alpha}, \quad\left\{\sqrt{\varepsilon} \alpha \partial_{x x x}^{3} u_{\varepsilon, \alpha}\right\}_{\varepsilon, \alpha} .
\end{aligned}
$$

If (2.6) and (2.12) are satisfied, then only ii) and iii) hold. 
Proof. We begin by assuming (2.5) and (2.11). Let $\lambda, \mu>0$ be two numbers that will be specified later. Multiplying (2.3) by $u_{\varepsilon, \alpha}^{3}+\lambda \partial_{t} u_{\varepsilon, \alpha}-\mu \partial_{x x}^{2} u_{\varepsilon, \alpha}$ and integrating the result over $\mathbb{R}$ we get

$$
\begin{aligned}
& \underbrace{\int_{\mathbb{R}} \partial_{t} u_{\varepsilon, \alpha} u_{\varepsilon, \alpha}^{3} d x}_{I_{1}(t)}+\underbrace{\lambda \int_{\mathbb{R}}\left(\partial_{t} u_{\varepsilon, \alpha}\right)^{2} d x}_{I_{2}(t)}-\underbrace{\mu \int_{\mathbb{R}} \partial_{t} u_{\varepsilon, \alpha} \partial_{x x}^{2} u_{\varepsilon, \alpha} d x}_{I_{3}(t)} \\
& -\underbrace{\alpha \int_{\mathbb{R}} \partial_{t x x}^{3} u_{\varepsilon, \alpha} u_{\varepsilon, \alpha}^{3} d x}_{I_{4}(t)}-\underbrace{\alpha \lambda \int_{\mathbb{R}} \partial_{t x x}^{3} u_{\varepsilon, \alpha} \partial_{t} u_{\varepsilon, \alpha} d x}_{I_{5}(t)} \\
& +\underbrace{\alpha \mu \int_{\mathbb{R}} \partial_{t x x}^{3} u_{\varepsilon, \alpha} \partial_{x x}^{2} u_{\varepsilon, \alpha} d x}_{I_{6}(t)}+\underbrace{\int_{\mathbb{R}} f^{\prime}\left(u_{\varepsilon, \alpha}\right) \partial_{x} u_{\varepsilon, \alpha} u_{\varepsilon, \alpha}^{3} d x}_{I_{7}(t)} \\
& +\underbrace{\lambda \int_{\mathbb{R}} f^{\prime}\left(u_{\varepsilon, \alpha}\right) \partial_{x} u_{\varepsilon, \alpha} \partial_{t} u_{\varepsilon, \alpha} d x}_{I_{8}(t)}-\underbrace{\mu \int_{\mathbb{R}} f^{\prime}\left(u_{\varepsilon, \alpha}\right) \partial_{x} u_{\varepsilon, \alpha} \partial_{x x}^{2} u_{\varepsilon, \alpha} d x}_{I_{9}(t)} \\
& =\underbrace{2 \alpha \int_{\mathbb{R}} \partial_{x} u_{\varepsilon, \alpha} \partial_{x x}^{2} u_{\varepsilon, \alpha} u_{\varepsilon, \alpha}^{3} d x}_{I_{10}(t)}+\underbrace{2 \alpha \lambda \int_{\mathbb{R}} \partial_{x} u_{\varepsilon, \alpha} \partial_{x x}^{2} u_{\varepsilon, \alpha} \partial_{t} u_{\varepsilon, \alpha} d x}_{I_{11}(t)} \\
& -\underbrace{2 \alpha \mu \int_{\mathbb{R}} \partial_{x} u_{\varepsilon, \alpha}\left(\partial_{x x}^{2} u_{\varepsilon, \alpha}\right)^{2} d x}_{I_{12}(t)}+\underbrace{\alpha \int_{\mathbb{R}} u_{\varepsilon, \alpha}^{4} \partial_{x x x}^{3} u_{\varepsilon, \alpha} d x}_{I_{13}(t)} \\
& +\underbrace{\alpha \lambda \int_{\mathbb{R}} u_{\varepsilon, \alpha} \partial_{t} u_{\varepsilon, \alpha} \partial_{x x x}^{3} u_{\varepsilon, \alpha} d x}_{I_{14}(t)}-\underbrace{\alpha \mu \int_{\mathbb{R}} u_{\varepsilon, \alpha} \partial_{x x}^{2} u_{\varepsilon, \alpha} \partial_{x x x}^{3} u_{\varepsilon, \alpha} d x}_{I_{15}(t)} \\
& +\underbrace{\varepsilon \int_{\mathbb{R}} u_{\varepsilon, \alpha}^{3} \partial_{x x}^{2} u_{\varepsilon, \alpha} d x}_{I_{16}(t)}+\underbrace{\varepsilon \lambda \int_{\mathbb{R}} \partial_{t} u_{\varepsilon, \alpha} \partial_{x x}^{2} u_{\varepsilon, \alpha} d x}_{I_{17}(t)}-\underbrace{\varepsilon \mu \int_{\mathbb{R}}\left(\partial_{x x}^{2} u_{\varepsilon, \alpha}\right)^{2} d x}_{I_{18}(t)} \\
& -\underbrace{\varepsilon \alpha \int_{\mathbb{R}} u_{\varepsilon, \alpha}^{3} \partial_{x x x x}^{4} u_{\varepsilon, \alpha} d x}_{I_{19}(t)}-\underbrace{\varepsilon \alpha \lambda \int_{\mathbb{R}} \partial_{t} u_{\varepsilon, \alpha} \partial_{x x x x}^{4} u_{\varepsilon, \alpha} d x}_{I_{20}(t)} \\
& +\underbrace{\varepsilon \alpha \mu \int_{\mathbb{R}} \partial_{x x}^{2} u_{\varepsilon, \alpha} \partial_{x x x x}^{4} u_{\varepsilon, \alpha} d x}_{I_{21}(t)} .
\end{aligned}
$$

Notice that $I_{7}(t)=0$ for all $t$. We rewrite the remaining terms in (4.7) as follows:

$$
\begin{aligned}
& I_{1}(t)-I_{3}(t)+I_{6}(t)-I_{17}(t)+I_{20}(t) \\
& =\frac{d}{d t} \int_{\mathbb{R}}\left(\frac{u_{\varepsilon, \alpha}^{4}}{4}+\frac{\mu+\varepsilon \lambda}{2}\left(\partial_{x} u_{\varepsilon, \alpha}\right)^{2}+\frac{\alpha(\mu+\varepsilon \lambda)}{2}\left(\partial_{x x}^{2} u_{\varepsilon, \alpha}\right)^{2}\right) d x, \\
& I_{2}(t)-I_{5}(t)-I_{16}(t)+I_{18}(t)-I_{21}(t) \\
& =\lambda \int_{\mathbb{R}}\left(\partial_{t} u_{\varepsilon, \alpha}\right)^{2} d x+\alpha \lambda \int_{\mathbb{R}}\left(\partial_{t x}^{2} u_{\varepsilon, \alpha}\right)^{2} d x+3 \varepsilon \int_{\mathbb{R}} u_{\varepsilon, \alpha}^{2}\left(\partial_{x} u_{\varepsilon, \alpha}\right)^{2} d x \\
& \quad+\varepsilon \mu \int_{\mathbb{R}}\left(\partial_{x x}^{2} u_{\varepsilon, \alpha}\right)^{2} d x+\varepsilon \alpha \mu \int_{\mathbb{R}}\left(\partial_{x x x}^{3} u_{\varepsilon, \alpha}\right)^{2} d x,
\end{aligned}
$$


and finally, using (4.2),

$$
\begin{aligned}
-I_{19} & =3 \varepsilon \alpha \int_{\mathbb{R}} u_{\varepsilon, \alpha}^{2} \partial_{x} u_{\varepsilon, \alpha} \partial_{x x x}^{3} u_{\varepsilon, \alpha} d x \\
& =-3 \varepsilon \alpha \int_{\mathbb{R}} u_{\varepsilon, \alpha}^{2}\left(\partial_{x x}^{2} u_{\varepsilon, \alpha}\right)^{2} d x-6 \varepsilon \alpha \int_{\mathbb{R}} u\left(\partial_{x} u_{\varepsilon, \alpha}\right)^{2} \partial_{x x}^{2} u_{\varepsilon, \alpha} d x \\
& =-3 \varepsilon \alpha \int_{\mathbb{R}} u_{\varepsilon, \alpha}^{2}\left(\partial_{x x}^{2} u_{\varepsilon, \alpha}\right)^{2} d x+2 \varepsilon \alpha \int_{\mathbb{R}}\left(\partial_{x} u_{\varepsilon, \alpha}\right)^{4} d x \\
& \leq-3 \varepsilon \alpha \int_{\mathbb{R}} u_{\varepsilon, \alpha}^{2}\left(\partial_{x x}^{2} u_{\varepsilon, \alpha}\right)^{2} d x+2 c_{1} \varepsilon \alpha \int_{\mathbb{R}} u_{\varepsilon, \alpha}^{2} d x \int_{\mathbb{R}}\left(\partial_{x x}^{2} u_{\varepsilon, \alpha}\right)^{2} d x \\
& \leq-3 \varepsilon \alpha \int_{\mathbb{R}} u_{\varepsilon, \alpha}^{2}\left(\partial_{x x}^{2} u_{\varepsilon, \alpha}\right)^{2} d x+2 c_{1} C_{0}^{2} \varepsilon \alpha \int_{\mathbb{R}}\left(\partial_{x x}^{2} u_{\varepsilon, \alpha}\right)^{2} d x,
\end{aligned}
$$

for some constant $c_{1}>0$.

Hence, using (4.8), (4.9), and (4.10), we can write (4.7) in the form

$$
\begin{aligned}
& \frac{d}{d t} \int_{\mathbb{R}}\left(\frac{u_{\varepsilon, \alpha}^{4}}{4}+\frac{\mu+\varepsilon \lambda}{2}\left(\partial_{x} u_{\varepsilon, \alpha}\right)^{2}+\frac{\alpha(\mu+\varepsilon \lambda)}{2}\left(\partial_{x x}^{2} u_{\varepsilon, \alpha}\right)^{2}\right) d x \\
& \quad+\lambda \int_{\mathbb{R}}\left(\partial_{t} u_{\varepsilon, \alpha}\right)^{2} d x+\alpha \lambda \int_{\mathbb{R}}\left(\partial_{t x}^{2} u_{\varepsilon, \alpha}\right)^{2} d x+3 \varepsilon \int_{\mathbb{R}} u_{\varepsilon, \alpha}^{2}\left(\partial_{x} u_{\varepsilon, \alpha}\right)^{2} d x \\
& \quad+\varepsilon \mu \int_{\mathbb{R}}\left(\partial_{x x}^{2} u_{\varepsilon, \alpha}\right)^{2} d x+\varepsilon \alpha \mu \int_{\mathbb{R}}\left(\partial_{x x x}^{3} u_{\varepsilon, \alpha}\right)^{2} d x \\
& \quad+3 \varepsilon \alpha \int_{\mathbb{R}} u_{\varepsilon, \alpha}^{2}\left(\partial_{x x}^{2} u_{\varepsilon, \alpha}\right)^{2} d x-2 c_{1} C_{0}^{2} \varepsilon \alpha \int_{\mathbb{R}}\left(\partial_{x x}^{2} u_{\varepsilon, \alpha}\right)^{2} d x \\
& \leq \alpha \int_{\mathbb{R}} \partial_{t x x}^{3} u_{\varepsilon, \alpha} u_{\varepsilon, \alpha}^{3} d x-\lambda \int_{\mathbb{R}} f^{\prime}\left(u_{\varepsilon, \alpha}\right) \partial_{x} u_{\varepsilon, \alpha} \partial_{t} u_{\varepsilon, \alpha} d x \\
& \quad+\mu \int_{\mathbb{R}} f^{\prime}\left(u_{\varepsilon, \alpha}\right) \partial_{x} u \partial_{x x}^{2} u d x+2 \alpha \int_{\mathbb{R}} \partial_{x} u_{\varepsilon, \alpha} \partial_{x x}^{2} u_{\varepsilon, \alpha} u_{\varepsilon, \alpha}^{3} d x \\
& \quad+2 \alpha \lambda \int_{\mathbb{R}} \partial_{x} u_{\varepsilon, \alpha} \partial_{x x}^{2} u_{\varepsilon, \alpha} \partial_{t} u_{\varepsilon, \alpha} d x-2 \alpha \mu \int_{\mathbb{R}} \partial_{x} u_{\varepsilon, \alpha}\left(\partial_{x x}^{2} u_{\varepsilon, \alpha}\right)^{2} d x \\
& \quad+\alpha \int_{\mathbb{R}} u_{\varepsilon, \alpha}^{4} \partial_{x x x}^{3} u_{\varepsilon, \alpha} d x+\alpha \lambda \int_{\mathbb{R}} u_{\varepsilon, \alpha} \partial_{t} u_{\varepsilon, \alpha} \partial_{x x x}^{3} u_{\varepsilon, \alpha} d x \\
& \quad-\alpha \mu \int_{\mathbb{R}} u_{\varepsilon, \alpha} \partial_{x x}^{2} u_{\varepsilon, \alpha} \partial_{x x x}^{3} u_{\varepsilon, \alpha} d x \\
& =J_{1}(t)+J_{2}(t)+J_{3}(t)+J_{4}(t)+J_{5}(t)+J_{6}(t)
\end{aligned}
$$

where

$$
\begin{aligned}
J_{1}(t) & :=2 \alpha \int_{\mathbb{R}} \partial_{x} u_{\varepsilon, \alpha} \partial_{x x}^{2} u_{\varepsilon, \alpha} u_{\varepsilon, \alpha}^{3} d x+\alpha \int_{\mathbb{R}} u_{\varepsilon, \alpha}^{4} \partial_{x x x}^{3} u_{\varepsilon, \alpha} d x, \\
J_{2}(t) & :=-2 \alpha \mu \int_{\mathbb{R}} \partial_{x} u_{\varepsilon, \alpha}\left(\partial_{x x}^{2} u_{\varepsilon, \alpha}\right)^{2} d x-\alpha \mu \int_{\mathbb{R}} u_{\varepsilon, \alpha} \partial_{x x}^{2} u_{\varepsilon, \alpha} \partial_{x x x}^{3} u_{\varepsilon, \alpha} d x, \\
J_{3}(t) & :=\alpha \int_{\mathbb{R}} \partial_{t x x}^{3} u_{\varepsilon, \alpha} u_{\varepsilon, \alpha}^{3} d x, \\
J_{4}(t) & :=-\lambda \int_{\mathbb{R}} f^{\prime}\left(u_{\varepsilon, \alpha}\right) \partial_{x} u_{\varepsilon, \alpha} \partial_{t} u_{\varepsilon, \alpha} d x, \\
J_{5}(t) & :=\mu \int_{\mathbb{R}} f^{\prime}\left(u_{\varepsilon, \alpha}\right) \partial_{x} u_{\varepsilon, \alpha} \partial_{x x}^{2} u_{\varepsilon, \alpha} d x, \\
J_{6}(t) & :=2 \alpha \lambda \int_{\mathbb{R}} \partial_{x} u_{\varepsilon, \alpha} \partial_{x x}^{2} u_{\varepsilon, \alpha} \partial_{t} u_{\varepsilon, \alpha} d x+\alpha \lambda \int_{\mathbb{R}} u_{\varepsilon, \alpha} \partial_{t} u_{\varepsilon, \alpha} \partial_{x x x}^{3} u_{\varepsilon, \alpha} d x .
\end{aligned}
$$


Now we estimate the terms $J_{1}, \ldots, J_{6}$ :

$$
\begin{aligned}
& J_{1}(t)=-2 \alpha \int_{\mathbb{R}} \partial_{x} u_{\varepsilon, \alpha} \partial_{x x}^{2} u_{\varepsilon, \alpha} u_{\varepsilon, \alpha}^{3} d x \\
& \leq \frac{\alpha}{\varepsilon} \int_{\mathbb{R}} u_{\varepsilon, \alpha}^{4}\left(\partial_{x} u_{\varepsilon, \alpha}\right)^{2} d x+\alpha \varepsilon \int_{\mathbb{R}} u_{\varepsilon, \alpha}^{2}\left(\partial_{x x}^{2} u_{\varepsilon, \alpha}\right)^{2} d x \\
& \leq \frac{2 C_{0}^{2} \sqrt{\alpha}}{\varepsilon} \int_{\mathbb{R}} u_{\varepsilon, \alpha}^{2}\left(\partial_{x} u_{\varepsilon, \alpha}\right)^{2} d x+\alpha \varepsilon \int_{\mathbb{R}} u_{\varepsilon, \alpha}^{2}\left(\partial_{x x}^{2} u_{\varepsilon, \alpha}\right)^{2} d x, \\
& J_{2}(t)=3 \alpha \mu \int_{\mathbb{R}} u_{\varepsilon, \alpha} \partial_{x x}^{2} u_{\varepsilon, \alpha} \partial_{x x x}^{3} u_{\varepsilon, \alpha} d x \\
& \leq \frac{9 \alpha \mu}{2 \varepsilon} \int_{\mathbb{R}} u_{\varepsilon, \alpha}^{2}\left(\partial_{x x}^{2} u_{\varepsilon, \alpha}\right)^{2} d x+\frac{\alpha \mu \varepsilon}{2} \int_{\mathbb{R}}\left(\partial_{x x x}^{3} u_{\varepsilon, \alpha}\right)^{2} d x, \\
& J_{3}(t)=-3 \alpha \int_{\mathbb{R}} \partial_{t x}^{2} u_{\varepsilon, \alpha} \partial_{x} u_{\varepsilon, \alpha} u_{\varepsilon, \alpha}^{2} d x \\
& \leq \frac{\alpha \lambda}{6} \int_{\mathbb{R}}\left(\partial_{t x}^{2} u_{\varepsilon, \alpha}\right)^{2} d x+\frac{27 \alpha}{2 \lambda} \int_{\mathbb{R}} u_{\varepsilon, \alpha}^{4}\left(\partial_{x} u_{\varepsilon, \alpha}\right)^{2} d x \\
& \leq \frac{\alpha \lambda}{6} \int_{\mathbb{R}}\left(\partial_{t x}^{2} u_{\varepsilon, \alpha}\right)^{2} d x+\frac{27 C_{0}^{2} \sqrt{\alpha}}{\lambda} \int_{\mathbb{R}} u_{\varepsilon, \alpha}^{2}\left(\partial_{x} u_{\varepsilon, \alpha}\right)^{2} d x, \\
& J_{4}(t) \leq \kappa_{0} \lambda \int_{\mathbb{R}}\left|u_{\varepsilon, \alpha} \partial_{x} u_{\varepsilon, \alpha} \partial_{t} u_{\varepsilon, \alpha}\right| d x \\
& \leq \frac{\lambda}{2} \int_{\mathbb{R}}\left(\partial_{t} u_{\varepsilon, \alpha}\right)^{2} d x+\frac{\kappa_{0}^{2} \lambda}{2} \int_{\mathbb{R}} u_{\varepsilon, \alpha}^{2}\left(\partial_{x} u_{\varepsilon, \alpha}\right)^{2} d x, \\
& J_{5}(t) \leq \kappa_{0} \mu \int_{\mathbb{R}}\left|u_{\varepsilon, \alpha} \partial_{x} u_{\varepsilon, \alpha} \partial_{x x}^{2} u_{\varepsilon, \alpha}\right| d x \\
& \leq \frac{\kappa_{0} \mu}{2 \varepsilon} \int_{\mathbb{R}} u_{\varepsilon, \alpha}^{2}\left(\partial_{x} u_{\varepsilon, \alpha}\right)^{2} d x+\frac{\kappa_{0} \mu \varepsilon}{2} \int_{\mathbb{R}}\left(\partial_{x x}^{2} u_{\varepsilon, \alpha}\right)^{2} d x, \\
& J_{6}(t)=\alpha \lambda \int_{\mathbb{R}} \partial_{t} u_{\varepsilon, \alpha} \partial_{x} u_{\varepsilon, \alpha} \partial_{x x}^{2} u_{\varepsilon, \alpha} d x-\alpha \lambda \int_{\mathbb{R}} u_{\varepsilon, \alpha} \partial_{t x}^{2} u_{\varepsilon, \alpha} \partial_{x x}^{2} u_{\varepsilon, \alpha} d x \\
& =-\frac{\alpha \lambda}{2} \int_{\mathbb{R}} \partial_{t x}^{2} u_{\varepsilon, \alpha}\left(\partial_{x} u_{\varepsilon, \alpha}\right)^{2} d x-\alpha \lambda \int_{\mathbb{R}} u_{\varepsilon, \alpha} \partial_{t x}^{2} u_{\varepsilon, \alpha} \partial_{x x}^{2} u_{\varepsilon, \alpha} d x \\
& \leq \frac{\alpha \lambda}{6} \int_{\mathbb{R}}\left(\partial_{t x}^{2} u_{\varepsilon, \alpha}\right)^{2} d x+\frac{3 \alpha \lambda}{2} \int_{\mathbb{R}}\left(\partial_{x} u_{\varepsilon, \alpha}\right)^{4} d x \\
& +\frac{\alpha \lambda}{6} \int_{\mathbb{R}}\left(\partial_{t x}^{2} u_{\varepsilon, \alpha}\right)^{2} d x+\frac{3 \alpha \lambda}{2} \int_{\mathbb{R}} u_{\varepsilon, \alpha}^{2}\left(\partial_{x x}^{2} u_{\varepsilon, \alpha}\right)^{2} d x \\
& \leq \frac{\alpha \lambda}{3} \int_{\mathbb{R}}\left(\partial_{t x}^{2} u_{\varepsilon, \alpha}\right)^{2} d x \\
& +\frac{3 c_{1} \alpha \lambda}{2} \int_{\mathbb{R}} u_{\varepsilon, \alpha}^{2} d x \int_{\mathbb{R}}\left(\partial_{x x}^{2} u_{\varepsilon, \alpha}\right)^{2} d x+\frac{3 \alpha \lambda}{2} \int_{\mathbb{R}} u_{\varepsilon, \alpha}^{2}\left(\partial_{x x}^{2} u_{\varepsilon, \alpha}\right)^{2} d x \\
& \leq \frac{\alpha \lambda}{3} \int_{\mathbb{R}}\left(\partial_{t x}^{2} u_{\varepsilon, \alpha}\right)^{2} d x+\frac{3 c_{1} C_{0}^{2} \alpha \lambda}{2} \int_{\mathbb{R}}\left(\partial_{x x}^{2} u_{\varepsilon, \alpha}\right)^{2} d x \\
& +\frac{3 \alpha \lambda}{2} \int_{\mathbb{R}} u_{\varepsilon, \alpha}^{2}\left(\partial_{x x}^{2} u_{\varepsilon, \alpha}\right)^{2} d x .
\end{aligned}
$$

We used (4.4) to estimate $J_{1}(t), J_{3}(t)$ and $(2.5)$ to estimate $J_{4}(t), J_{5}(t)$. To estimate $J_{6}(t)$ we used the inequality

$$
\int_{\mathbb{R}}\left(\partial_{x} u_{\varepsilon, \alpha}\right)^{4} d x \leq c_{1} \int_{\mathbb{R}} u_{\varepsilon, \alpha}^{2} d x \int_{\mathbb{R}}\left(\partial_{x x}^{2} u_{\varepsilon, \alpha}\right)^{2} d x,
$$

where $c_{1}$ is some universal constant. 
Substituting (4.12), (4.13), (4.14), (4.15), (4.16), (4.17) into (4.11), we get

$$
\begin{aligned}
& \frac{d}{d t} \int_{\mathbb{R}}\left(\frac{u_{\varepsilon, \alpha}^{4}}{4}+\frac{\mu+\varepsilon \lambda}{2}\left(\partial_{x} u_{\varepsilon, \alpha}\right)^{2}+\frac{\alpha(\mu+\varepsilon \lambda)}{2}\left(\partial_{x x}^{2} u_{\varepsilon, \alpha}\right)^{2}\right) d x \\
& \quad+\lambda \int_{\mathbb{R}}\left(\partial_{t} u_{\varepsilon, \alpha}\right)^{2} d x+\alpha \lambda \int_{\mathbb{R}}\left(\partial_{t x}^{2} u_{\varepsilon, \alpha}\right)^{2} d x+3 \varepsilon \int_{\mathbb{R}} u_{\varepsilon, \alpha}^{2}\left(\partial_{x} u_{\varepsilon, \alpha}\right)^{2} d x \\
& \quad+\varepsilon \mu \int_{\mathbb{R}}\left(\partial_{x x}^{2} u_{\varepsilon, \alpha}\right)^{2} d x+\varepsilon \alpha \mu \int_{\mathbb{R}}\left(\partial_{x x x}^{3} u_{\varepsilon, \alpha}\right)^{2} d x+3 \varepsilon \alpha \int_{\mathbb{R}} u_{\varepsilon, \alpha}^{2}\left(\partial_{x x}^{2} u_{\varepsilon, \alpha}\right)^{2} d x \\
& \leq \Lambda_{1} \int_{\mathbb{R}}\left(\partial_{x x}^{2} u_{\varepsilon, \alpha}\right)^{2} d x+\Lambda_{2} \int_{\mathbb{R}} u_{\varepsilon, \alpha}^{2}\left(\partial_{x} u_{\varepsilon, \alpha}\right)^{2} d x+\Lambda_{3} \int_{\mathbb{R}} u_{\varepsilon, \alpha}^{2}\left(\partial_{x x}^{2} u_{\varepsilon, \alpha}\right)^{2} d x \\
& \quad+\frac{\alpha \varepsilon \mu}{2} \int_{\mathbb{R}}\left(\partial_{x x x}^{3} u_{\varepsilon, \alpha}\right)^{2} d x+\frac{\alpha \lambda}{2} \int_{\mathbb{R}}\left(\partial_{t x}^{2} u_{\varepsilon, \alpha}\right)^{2} d x+\frac{\lambda}{2} \int_{\mathbb{R}}\left(\partial_{t} u_{\varepsilon, \alpha}\right)^{2},
\end{aligned}
$$

where

$$
\begin{aligned}
& \Lambda_{1}:=2 c_{1} C_{0}^{2} \varepsilon \alpha+\frac{3 c_{1} C_{0}^{2} \alpha \lambda}{2}+\frac{\kappa_{0} \mu \varepsilon}{2}, \\
& \Lambda_{2}:=\frac{2 C_{0}^{2} \sqrt{\alpha}}{\varepsilon}+\frac{27 C_{0}^{2} \sqrt{\alpha}}{\lambda}+\frac{\kappa_{0}^{2} \lambda}{2}+\frac{\kappa_{0} \mu}{2 \varepsilon}, \\
& \Lambda_{3}:=\alpha \varepsilon+\frac{9 \alpha \mu}{2 \varepsilon}+\frac{3 \alpha \lambda}{2} .
\end{aligned}
$$

Finally, choosing (see (2.11))

$$
\alpha \leq k_{1} \varepsilon^{4}, \quad \lambda=k_{2} \varepsilon, \quad \mu=k_{3} \alpha\left(\leq k_{1} k_{2} \varepsilon^{4}\right),
$$

for some constant $k_{1}, k_{2}, k_{3}>0$ (which are independent of $\varepsilon, \alpha$ ), we have

$$
\Lambda_{1}=O(\varepsilon \alpha)=O(\varepsilon \mu), \quad \Lambda_{2}=O(\varepsilon), \quad \Lambda_{3}=O(\varepsilon \alpha) .
$$

Hence, from (4.3),

$$
\Lambda_{1} \int_{0}^{t} \int_{\mathbb{R}}\left(\partial_{x x}^{2} u_{\varepsilon, \alpha}\right)^{2} d \tau d x \leq c_{2}
$$

for some constant $c_{2}>0$ depending only on $C_{0}$. Next, we can choose $k_{1}, k_{2}, k_{3}$ small enough (independently of $\varepsilon, \alpha$ ) such that

$$
3 \varepsilon-\Lambda_{2} \leq \frac{\varepsilon}{2}, \quad 3 \varepsilon \alpha-\Lambda_{3} \leq \frac{\varepsilon \alpha}{2} .
$$

Integrating (4.19) on the interval $(0, t)$, using (4.19), (4.21), and (4.22), yields

$$
\begin{aligned}
\int_{\mathbb{R}}( & \left.\frac{u_{\varepsilon, \alpha}^{4}}{4}+\frac{\mu+\varepsilon \lambda}{2}\left(\partial_{x} u_{\varepsilon, \alpha}\right)^{2}+\frac{\alpha(\mu+\varepsilon \lambda)}{2}\left(\partial_{x x}^{2} u_{\varepsilon, \alpha}\right)^{2}\right) d x \\
& +\frac{\lambda}{2} \int_{0}^{t} \int_{\mathbb{R}}\left(\partial_{t} u_{\varepsilon, \alpha}\right)^{2} d x d \tau+\frac{\alpha \lambda}{2} \int_{0}^{t} \int_{\mathbb{R}}\left(\partial_{t x}^{2} u_{\varepsilon, \alpha}\right)^{2} d x d \tau \\
& +\frac{\varepsilon}{2} \int_{0}^{t} \int_{\mathbb{R}} u_{\varepsilon, \alpha}^{2}\left(\partial_{x} u_{\varepsilon, \alpha}\right)^{2} d \tau d x+\varepsilon \mu \int_{0}^{T} \int_{\mathbb{R}}\left(\partial_{x x}^{2} u_{\varepsilon, \alpha}\right)^{2} d x d \tau \\
& +\frac{\varepsilon \alpha \mu}{2} \int_{0}^{t} \int_{\mathbb{R}}\left(\partial_{x x x}^{3} u_{\varepsilon, \alpha}\right)^{2} d x d \tau+\frac{\varepsilon \alpha}{2} \int_{0}^{t} \int_{\mathbb{R}} u_{\varepsilon, \alpha}^{2}\left(\partial_{x x}^{2} u_{\varepsilon, \alpha}\right)^{2} d x d \tau \leq c_{3},
\end{aligned}
$$

for some constant $c_{3}>0$ depending only on $C_{0}$. Thanks to (4.20) the proof is done.

We now turn to the case in which (2.6) and (2.12) hold. Since the proof is similar we simply sketch it. Let $\lambda, \mu>0$ be two numbers to be specified later. Multiplying (2.3) by $\lambda \partial_{t} u_{\varepsilon, \alpha}-\mu \partial_{x x}^{2} u_{\varepsilon, \alpha}$ 
and manipulating the terms as in (4.7), we find eventually

$$
\begin{aligned}
& \frac{d}{d t} \int_{\mathbb{R}}\left(\frac{\mu+\varepsilon \lambda}{2}\left(\partial_{x} u_{\varepsilon, \alpha}\right)^{2}+\frac{\alpha(\mu+\varepsilon \lambda)}{2}\left(\partial_{x x}^{2} u_{\varepsilon, \alpha}\right)^{2}\right) d x \\
& \quad+\lambda \int_{\mathbb{R}}\left(\partial_{t} u_{\varepsilon, \alpha}\right)^{2} d x+\alpha \lambda \int_{\mathbb{R}}\left(\partial_{t x}^{2} u_{\varepsilon, \alpha}\right)^{2} d x \\
& \quad+\varepsilon \mu \int_{\mathbb{R}}\left(\partial_{x x}^{2} u_{\varepsilon, \alpha}\right)^{2} d x+\varepsilon \alpha \mu \int_{\mathbb{R}}\left(\partial_{x x x}^{3} u_{\varepsilon, \alpha}\right)^{2} d x \\
& =-\lambda \int_{\mathbb{R}} f^{\prime}\left(u_{\varepsilon, \alpha}\right) \partial_{x} u_{\varepsilon, \alpha} \partial_{t} u_{\varepsilon, \alpha} d x+\mu \int_{\mathbb{R}} f^{\prime}\left(u_{\varepsilon, \alpha}\right) \partial_{x} u \partial_{x x}^{2} u d x \\
& \quad+2 \alpha \lambda \int_{\mathbb{R}} \partial_{x} u_{\varepsilon, \alpha} \partial_{x x}^{2} u_{\varepsilon, \alpha} \partial_{t} u_{\varepsilon, \alpha} d x-2 \alpha \mu \int_{\mathbb{R}} \partial_{x} u_{\varepsilon, \alpha}\left(\partial_{x x}^{2} u_{\varepsilon, \alpha}\right)^{2} d x \\
& \quad+\alpha \lambda \int_{\mathbb{R}} u_{\varepsilon, \alpha} \partial_{t} u_{\varepsilon, \alpha} \partial_{x x x}^{3} u_{\varepsilon, \alpha} d x-\alpha \mu \int_{\mathbb{R}} u_{\varepsilon, \alpha} \partial_{x x}^{2} u_{\varepsilon, \alpha} \partial_{x x x}^{3} u_{\varepsilon, \alpha} d x \\
& =J_{2}(t)+J_{7}(t)+J_{8}(t)+J_{6}(t),
\end{aligned}
$$

where

$$
\begin{aligned}
& J_{7}(t):=-\lambda \int_{\mathbb{R}} f^{\prime}\left(u_{\varepsilon, \alpha}\right) \partial_{x} u_{\varepsilon, \alpha} \partial_{t} u_{\varepsilon, \alpha} d x, \\
& J_{8}(t):=\mu \int_{\mathbb{R}} f^{\prime}\left(u_{\varepsilon, \alpha}\right) \partial_{x} u_{\varepsilon, \alpha} \partial_{x x}^{2} u_{\varepsilon, \alpha} d x .
\end{aligned}
$$

We can estimate the terms $J_{7}$ and $J_{8}$ in the following way:

$$
\begin{aligned}
J_{7}(t) & \leq \kappa_{2} \lambda \int_{\mathbb{R}}\left|\partial_{x} u_{\varepsilon, \alpha} \partial_{t} u_{\varepsilon, \alpha}\right| d x \\
& \leq \frac{\lambda}{2} \int_{\mathbb{R}}\left(\partial_{t} u_{\varepsilon, \alpha}\right)^{2} d x+\frac{\kappa_{2}^{2} \lambda}{2} \int_{\mathbb{R}}\left(\partial_{x} u_{\varepsilon, \alpha}\right)^{2} d x, \\
J_{8}(t) & \leq \kappa_{2} \mu \int_{\mathbb{R}}\left|\partial_{x} u_{\varepsilon, \alpha} \partial_{x x}^{2} u_{\varepsilon, \alpha}\right| d x \\
& \leq \frac{\kappa_{2} \mu}{2 \varepsilon} \int_{\mathbb{R}}\left(\partial_{x} u_{\varepsilon, \alpha}\right)^{2} d x+\frac{\kappa_{2} \mu \varepsilon}{2} \int_{\mathbb{R}}\left(\partial_{x x}^{2} u_{\varepsilon, \alpha}\right)^{2} d x .
\end{aligned}
$$

Substituting (4.12), (4.25), (4.26), (4.17) into (4.24), we get

$$
\begin{aligned}
& \frac{d}{d t} \int_{\mathbb{R}}\left(\frac{\mu+\varepsilon \lambda}{2}\left(\partial_{x} u_{\varepsilon, \alpha}\right)^{2}+\frac{\alpha(\mu+\varepsilon \lambda)}{2}\left(\partial_{x x}^{2} u_{\varepsilon, \alpha}\right)^{2}\right) d x \\
& \quad+\lambda \int_{\mathbb{R}}\left(\partial_{t} u_{\varepsilon, \alpha}\right)^{2} d x+\alpha \lambda \int_{\mathbb{R}}\left(\partial_{t x}^{2} u_{\varepsilon, \alpha}\right)^{2} d x \\
& \quad+\varepsilon \mu \int_{\mathbb{R}}\left(\partial_{x x}^{2} u_{\varepsilon, \alpha}\right)^{2} d x+\varepsilon \alpha \mu \int_{\mathbb{R}}\left(\partial_{x x x}^{3} u_{\varepsilon, \alpha}\right)^{2} d x \\
& \leq \Lambda_{4} \int_{\mathbb{R}}\left(\partial_{x x}^{2} u_{\varepsilon, \alpha}\right)^{2} d x+\Lambda_{5} \int_{\mathbb{R}}\left(\partial_{x} u_{\varepsilon, \alpha}\right)^{2} d x+\Lambda_{6} \int_{\mathbb{R}} u_{\varepsilon, \alpha}^{2}\left(\partial_{x x}^{2} u_{\varepsilon, \alpha}\right)^{2} d x \\
& \quad+\frac{\alpha \varepsilon \mu}{2} \int_{\mathbb{R}}\left(\partial_{x x x}^{3} u_{\varepsilon, \alpha}\right)^{2} d x+\frac{\alpha \lambda}{3} \int_{\mathbb{R}}\left(\partial_{t x}^{2} u_{\varepsilon, \alpha}\right)^{2} d x+\frac{\lambda}{2} \int_{\mathbb{R}}\left(\partial_{t} u_{\varepsilon, \alpha}\right)^{2},
\end{aligned}
$$

where

$$
\Lambda_{4}:=\frac{3 c_{1} C_{0}^{2} \alpha \lambda}{8}+\frac{\kappa_{2} \mu \varepsilon}{2}, \quad \Lambda_{5}:=\frac{\kappa_{2}^{2} \lambda}{2}+\frac{\kappa_{2} \mu}{2 \varepsilon}, \quad \Lambda_{6}:=\frac{9 \alpha \mu}{2 \varepsilon}+\frac{3 \alpha \lambda}{2} .
$$

Arguing as in the previous case and employing (2.12), choosing

$$
\alpha \leq k_{1} \varepsilon^{2}, \quad \lambda=k_{2} \varepsilon, \quad \mu=k_{3} \alpha\left(\leq k_{1} k_{2} \varepsilon^{2}\right),
$$

for some sufficiently small constants $k_{1}, k_{2}, k_{3}>0$ (which are independent of $\varepsilon, \alpha$ ), we have

$$
\Lambda_{1}=O(\varepsilon \alpha)=O(\varepsilon \mu), \quad \Lambda_{2}=O(\varepsilon), \quad \Lambda_{3}=O(\varepsilon \alpha) .
$$


Integrating (4.27) over the time interval $(0, t)$ yields

$$
\begin{aligned}
\int_{\mathbb{R}}( & \left.\frac{\mu+\varepsilon \lambda}{2}\left(\partial_{x} u_{\varepsilon, \alpha}\right)^{2}+\frac{\alpha(\mu+\varepsilon \lambda)}{2}\left(\partial_{x x}^{2} u_{\varepsilon, \alpha}\right)^{2}\right) d x \\
& +\frac{\lambda}{2} \int_{0}^{t} \int_{\mathbb{R}}\left(\partial_{t} u_{\varepsilon, \alpha}\right)^{2} d x d \tau+\frac{\alpha \lambda}{2} \int_{0}^{t} \int_{\mathbb{R}}\left(\partial_{t x}^{2} u_{\varepsilon, \alpha}\right)^{2} d x d \tau \\
& +\frac{\varepsilon}{2} \int_{0}^{t} \int_{\mathbb{R}} u_{\varepsilon, \alpha}^{2}\left(\partial_{x} u_{\varepsilon, \alpha}\right)^{2} d \tau d x+\varepsilon \mu \int_{0}^{T} \int_{\mathbb{R}}\left(\partial_{x x}^{2} u_{\varepsilon, \alpha}\right)^{2} d x d \tau \\
& +\frac{\varepsilon \alpha \mu}{2} \int_{0}^{t} \int_{\mathbb{R}}\left(\partial_{x x x}^{3} u_{\varepsilon, \alpha}\right)^{2} d x d \tau+\frac{\varepsilon \alpha}{2} \int_{0}^{t} \int_{\mathbb{R}} u_{\varepsilon, \alpha}^{2}\left(\partial_{x x}^{2} u_{\varepsilon, \alpha}\right)^{2} d x d \tau \leq c
\end{aligned}
$$

for some constant $c>0$ depending only on $C_{0}$. Thanks to (4.28) the proof is done.

\section{Proof of Theorem 2.1}

In this section we prove Theorem 2.1. The proof will be accomplished through Lemma 5.2 (convergence) and Lemma 5.3 (entropy satisfaction) below. We begin however with deriving a precise estimate on the entropy production, which eventually results in its $H_{\mathrm{loc}}^{-1}$ compactness.

A pair of functions $(\eta, q)$ is called an entropy-entropy flux pair if $\eta: \mathbb{R} \rightarrow \mathbb{R}$ is a $C^{2}$ function and $q: \mathbb{R} \rightarrow \mathbb{R}$ is defined by

$$
q(u)=\int_{0}^{u} \eta^{\prime}(\zeta) f^{\prime}(\zeta) d \zeta
$$

An entropy-entropy flux pair $(\eta, q)$ is called a convex/compactly supported if, in addition, $\eta$ is convex/compactly supported.

Lemma 5.1. Assume that (2.7)-(2.10) hold. If (2.5) and (2.11) or (2.6) and (2.12) are satisfied, then for any compactly supported entropy-entropy flux pair $(\eta, q)$,

$$
\partial_{t} \eta\left(u_{\varepsilon, \alpha}\right)+\partial_{x} q\left(u_{\varepsilon, \alpha}\right)=\mathcal{L}_{\varepsilon, \alpha}^{1}+\mathcal{L}_{\varepsilon, \alpha}^{2},
$$

where the distributions $\mathcal{L}_{\varepsilon, \alpha}^{1}, \mathcal{L}_{\varepsilon, \alpha}^{2}$ possess the following properties:

$$
\begin{aligned}
& \mathcal{L}_{\varepsilon, \alpha}^{1} \rightarrow 0 \text { in } H^{-1}\left(\mathbb{R}_{+} \times \mathbb{R}\right), \\
& \mathcal{L}_{\varepsilon, \alpha}^{2} \text { is uniformly bounded in } \mathcal{M}\left(\mathbb{R}_{+} \times \mathbb{R}\right) .
\end{aligned}
$$

Proof. First of all, notice that the constant

$$
\begin{aligned}
K_{0}:=\sup _{\varepsilon, \alpha}\{ & \left\|\sqrt{\varepsilon} \partial_{t} u_{\varepsilon, \alpha}\right\|_{L^{2}},\left\|\sqrt{\varepsilon \alpha} \partial_{t x}^{2} u_{\varepsilon, \alpha}\right\|_{L^{2}}, \\
& \left.\left\|\sqrt{\varepsilon} u_{\varepsilon, \alpha} \partial_{x} u_{\varepsilon, \alpha}\right\|_{L^{2}},\left\|\sqrt{\varepsilon \alpha} u_{\varepsilon, \alpha} \partial_{x x}^{2} u_{\varepsilon, \alpha}\right\|_{L^{2}},\left\|\alpha \sqrt{\varepsilon} \partial_{x x x}^{3} u_{\varepsilon, \alpha}\right\|_{L^{2}}\right\}
\end{aligned}
$$

is finite thanks to Lemma 4.2 .

Writing the nonlinear dispersive terms on the right-hand side of (2.3) as

$$
2 \alpha \partial_{x} u_{\varepsilon, \alpha} \partial_{x x}^{2} u_{\varepsilon, \alpha}+\alpha u_{\varepsilon, \alpha} \partial_{x x x}^{3} u_{\varepsilon, \alpha}=\frac{\alpha}{2} \partial_{x}\left(\partial_{x} u_{\varepsilon, \alpha}\right)^{2}+\partial_{x}\left(\alpha u_{\varepsilon, \alpha} \partial_{x x}^{2} u_{\varepsilon, \alpha}\right)
$$

and introducing the function

$$
\Gamma_{\varepsilon, \alpha}:=\alpha \partial_{t x}^{2} u_{\varepsilon, \alpha}+\frac{\alpha}{2}\left(\partial_{x} u_{\varepsilon, \alpha}\right)^{2}+\alpha u_{\varepsilon, \alpha} \partial_{x x}^{2} u_{\varepsilon, \alpha}+\varepsilon \partial_{x} u_{\varepsilon, \alpha}-\varepsilon \alpha \partial_{x x x}^{3} u_{\varepsilon, \alpha},
$$

multiplying (2.3) by $\eta^{\prime}\left(u_{\varepsilon, \alpha}\right)$ yields

$$
\begin{aligned}
& \partial_{t} \eta\left(u_{\varepsilon, \alpha}\right)+\partial_{x} q\left(u_{\varepsilon, \alpha}\right) \\
& =\left(\alpha \partial_{t x x}^{3} u_{\varepsilon, \alpha}+2 \alpha \partial_{x} u_{\varepsilon, \alpha} \partial_{x x}^{2} u_{\varepsilon, \alpha}+\alpha u_{\varepsilon, \alpha} \partial_{x x x}^{3} u_{\varepsilon, \alpha}\right. \\
& \left.\quad+\varepsilon \partial_{x x}^{2} u_{\varepsilon, \alpha}-\varepsilon \alpha \partial_{x x x x}^{4} u_{\varepsilon, \alpha}\right) \eta^{\prime}\left(u_{\varepsilon, \alpha}\right) \\
& =\eta^{\prime}\left(u_{\varepsilon, \alpha}\right) \partial_{x} \Gamma_{\varepsilon, \alpha} \\
& =\partial_{x}\left(\eta^{\prime}\left(u_{\varepsilon, \alpha}\right) \Gamma_{\varepsilon, \alpha}\right)-\eta^{\prime \prime}\left(u_{\varepsilon, \alpha}\right) \partial_{x} u_{\varepsilon, \alpha} \Gamma_{\varepsilon, \alpha}
\end{aligned}
$$


We claim that

$$
\begin{aligned}
& \Gamma_{\varepsilon, \alpha} \rightarrow 0 \text { strongly } L^{2}\left(\mathbb{R}_{+} \times \mathbb{R}\right) \text { as } \varepsilon, \alpha \rightarrow 0+, \\
& \partial_{x} u_{\varepsilon, \alpha} \Gamma_{\varepsilon, \alpha} \text { is uniformly bounded in } L^{1}\left(\mathbb{R}_{+} \times \mathbb{R}\right),
\end{aligned}
$$

which immediately proves the lemma.

In view of (4.18) and (4.2), the following inequality holds:

$$
\begin{aligned}
\int_{\mathbb{R}_{+}} \int_{\mathbb{R}}\left(\partial_{x} u_{\varepsilon, \alpha}\right)^{4} d x d t & \leq c_{1} \int_{\mathbb{R}_{+}}\left(\int_{\mathbb{R}} u_{\varepsilon, \alpha}^{2} d x\right)\left(\int_{\mathbb{R}}\left(\partial_{x x}^{2} u_{\varepsilon, \alpha}\right)^{2} d x\right) d t \\
& \leq c_{1} C_{0}^{2} \int_{\mathbb{R}_{+}} \int_{\mathbb{R}}\left(\partial_{x x}^{2} u_{\varepsilon, \alpha}\right)^{2} d x d t .
\end{aligned}
$$

Using this inequality, (2.11) or (2.12), (4.3), and (5.1), we obtain

$$
\begin{aligned}
& \left\|\Gamma_{\varepsilon, \alpha}\right\|_{L^{2}\left(\mathbb{R}_{+} \times \mathbb{R}\right)} \\
& \leq\left\|\alpha \partial_{t x}^{2} u_{\varepsilon, \alpha}\right\|_{L^{2}\left(\mathbb{R}_{+} \times \mathbb{R}\right)}+\frac{1}{2}\left\|\alpha\left(\partial_{x} u_{\varepsilon, \alpha}\right)^{2}\right\|_{L^{2}\left(\mathbb{R}_{+} \times \mathbb{R}\right)} \\
& \quad+\left\|\alpha u_{\varepsilon, \alpha} \partial_{x x}^{2} u_{\varepsilon, \alpha}\right\|_{L^{2}\left(\mathbb{R}_{+} \times \mathbb{R}\right)}+\left\|\varepsilon \partial_{x} u_{\varepsilon, \alpha}\right\|_{L^{2}}+\left\|\varepsilon \alpha \partial_{x x x}^{3} u_{\varepsilon, \alpha}\right\|_{L^{2}\left(\mathbb{R}_{+} \times \mathbb{R}\right)} \\
& \leq \sqrt{\frac{\alpha}{\varepsilon}}\left\|\sqrt{\varepsilon \alpha} \partial_{t x}^{2} u_{\varepsilon, \alpha}\right\|_{L^{2}\left(\mathbb{R}_{+} \times \mathbb{R}\right)}+\frac{\sqrt{c_{1}} C_{0}}{2} \sqrt{\frac{\alpha}{\varepsilon}}\left\|\sqrt{\varepsilon \alpha} \partial_{x x}^{2} u_{\varepsilon, \alpha}\right\|_{L^{2}\left(\mathbb{R}_{+} \times \mathbb{R}\right)} \\
& \quad+\sqrt{\frac{\alpha}{\varepsilon}}\left\|\sqrt{\varepsilon \alpha} u_{\varepsilon, \alpha} \partial_{x x}^{2} u_{\varepsilon, \alpha}\right\|_{L^{2}}+\sqrt{\varepsilon}\left\|\sqrt{\varepsilon} \partial_{x} u_{\varepsilon, \alpha}\right\|_{L^{2}}+\sqrt{\varepsilon}\left\|\alpha \sqrt{\varepsilon} \partial_{x x x}^{3} u_{\varepsilon, \alpha}\right\|_{L^{2}\left(\mathbb{R}_{+} \times \mathbb{R}\right)} \\
& \leq \sqrt{\frac{\alpha}{\varepsilon}} K_{0}+\frac{\sqrt{c_{1}} C_{0}^{2}}{2 \sqrt{2}} \sqrt{\frac{\alpha}{\varepsilon}}+\sqrt{\frac{\alpha}{\varepsilon}} K_{0}+\sqrt{\varepsilon} \frac{C_{0}}{\sqrt{2}}+\sqrt{\varepsilon} K_{0}=O\left(\sqrt{\frac{\alpha}{\varepsilon}}+\sqrt{\varepsilon}\right),
\end{aligned}
$$

which clearly proves (5.4). Moreover, using Hölder's inequality, (4.3), and (2.11) or (2.12),

$$
\begin{aligned}
& \left\|\partial_{x} u_{\varepsilon, \alpha} \Gamma_{\varepsilon, \alpha}\right\|_{L^{1}\left(\mathbb{R}_{+} \times \mathbb{R}\right)} \\
& \leq\left\|\partial_{x} u_{\varepsilon, \alpha}\right\|_{L^{2}\left(\mathbb{R}_{+} \times \mathbb{R}\right)}\left\|\Gamma_{\varepsilon, \alpha}\right\|_{L^{2}\left(\mathbb{R}_{+} \times \mathbb{R}\right)} \\
& \leq \frac{C_{0}}{\sqrt{2 \varepsilon}}\left(\sqrt{\frac{\alpha}{\varepsilon}} K_{0}+\frac{\sqrt{c_{1}} C_{0}^{2}}{2 \sqrt{2}} \sqrt{\frac{\alpha}{\varepsilon}}+\sqrt{\frac{\alpha}{\varepsilon}} K_{0}+\sqrt{\varepsilon} \frac{C_{0}}{\sqrt{2}}+\sqrt{\varepsilon} K_{0}\right) \\
& =\frac{C_{0}}{\sqrt{2}}\left(\frac{\sqrt{\alpha}}{\varepsilon} K_{0}+\frac{\sqrt{c_{1}} C_{0}^{2}}{2 \sqrt{2}} \frac{\sqrt{\alpha}}{\varepsilon}+\frac{\sqrt{\alpha}}{\varepsilon} K_{0}+\frac{C_{0}}{\sqrt{2}}+K_{0}\right)=O(1),
\end{aligned}
$$

and this proves (5.5).

Lemma 5.2. Assume that (2.7)-(2.10) hold.

i) If (2.5) and (2.11) are satisfied, then there exist two sequences $\left\{\varepsilon_{k}\right\}_{k \in \mathbb{N}},\left\{\alpha_{k}\right\}_{k \in \mathbb{N}}$, with $\varepsilon_{k}, \alpha_{k} \rightarrow 0$, and a limit function $u \in L^{\infty}\left(\mathbb{R}_{+} ; L^{4}(\mathbb{R}) \cap L^{2}(\mathbb{R})\right)$, such that

$u_{\varepsilon_{k}, \alpha_{k}} \rightarrow u$ strongly in $L_{\text {loc }}^{p}\left(\mathbb{R}_{+} \times \mathbb{R}\right)$, for each $1 \leq p<4$.

ii) If (2.6) and (2.12) are satisfied, then there exist two sequences $\left\{\varepsilon_{k}\right\}_{k \in \mathbb{N}},\left\{\alpha_{k}\right\}_{k \in \mathbb{N}}$, with $\varepsilon_{k}, \alpha_{k} \rightarrow 0$, and a limit function $u \in L^{\infty}\left(\mathbb{R}_{+} ; L^{2}(\mathbb{R})\right)$, such that

$u_{\varepsilon_{k}, \alpha_{k}} \rightarrow u$ strongly in $L_{\text {loc }}^{p}\left(\mathbb{R}_{+} \times \mathbb{R}\right)$, for each $1 \leq p<2$.

Moreover, in both cases the limit function $u$ is a weak solution of (2.1), (2.2):

$$
\int_{\mathbb{R}_{+}} \int_{\mathbb{R}}\left(u \partial_{t} \phi+f(u) \partial_{x} \phi\right) d x d t+\int_{\mathbb{R}} u_{0}(x) \phi(0, x) d x=0, \quad \forall \phi \in C_{c}^{\infty}([0, \infty) \times \mathbb{R}) .
$$

Proof. Let $(\eta, q)$ be a compactly supported entropy-entropy flux pair. According to Lemma 5.1, $\partial_{t} \eta\left(u_{\varepsilon, \alpha}\right)+\partial_{x} q\left(u_{\varepsilon, \alpha}\right)$ equals one term that tends to zero in $H^{-1}\left(\mathbb{R}_{+} \times \mathbb{R}\right)$ plus another one that is uniformly bounded in the sense of measures on $\mathbb{R}_{+} \times \mathbb{R}$. In addition, since $\eta$ is compactly supported, it is immediate that $\partial_{t} \eta\left(u_{\varepsilon, \alpha}\right)+\partial_{x} q\left(u_{\varepsilon, \alpha}\right)$ is uniformly bounded in $W^{-1, \infty}\left(\mathbb{R}_{+} \times \mathbb{R}\right)$. Therefore, by Lemma 3.2, the sequence of distributions $\left\{\partial_{t} \eta\left(u_{\varepsilon, \alpha}\right)+\partial_{x} q\left(u_{\varepsilon, \alpha}\right)\right\}_{\varepsilon, \alpha}$ belongs to compact subset 
of $H_{\text {loc }}^{-1}(\Omega)$, for fixed bounded open subset $\Omega$ of $\mathbb{R}_{+} \times \mathbb{R}$. Lemma 3.1 (with $s=1$ ) then yields the existence of a subsequence of $\left\{u_{\varepsilon, \alpha}\right\}_{\varepsilon, \alpha}$ that converges strongly in $L^{r}(\Omega)$ for any $1 \leq r<4$ in the first case and $1 \leq r<2$ in the second one, for each fixed bounded open set $\Omega \subset \mathbb{R}_{+} \times \mathbb{R}$. The first part of the lemma then follows by a standard diagonal argument. Using this strong convergence, the second part follows by passing to the limit in the weak formulation of (2.3), (2.4), see (5.3) with $\eta(u)=u, q(u)=f(u)$ and (5.4).

The next lemma shows that the limit function $u$ constructed in Lemma 5.2 dissipates the energy.

Lemma 5.3. Assume that (2.7), (2.9), (2.10), and (2.5)-(2.13) or (2.6)-(2.14) hold. Define $q: \mathbb{R} \rightarrow \mathbb{R}$ by $q(u):=\int_{0}^{u} \zeta f^{\prime}(\zeta) d \zeta$. Then the the limit function u constructed in Lemma 5.2 satisfies $\partial_{t}\left(\frac{u^{2}}{2}\right)+\partial_{x} q(u) \leq 0$ in the weak sense on $\mathbb{R}_{+} \times \mathbb{R}$.

Proof. Since $u_{\varepsilon, \alpha}$ is bounded in $L^{\infty}\left(\mathbb{R}_{+} ; L^{4}(\mathbb{R})\right)$ or in $L^{\infty}\left(\mathbb{R}_{+} ; L^{2}(\mathbb{R})\right)$, multiplying (2.3) by $u_{\varepsilon, \alpha}$ is meaningful and yields

$$
\partial_{t}\left(\frac{u_{\varepsilon, \alpha}^{2}}{2}\right)+\partial_{x} q\left(u_{\varepsilon, \alpha}\right)=\partial_{x}\left(u_{\varepsilon, \alpha} \Gamma_{\varepsilon, \alpha}\right)-\partial_{x} u_{\varepsilon, \alpha} \Delta_{\varepsilon, \alpha}-\varepsilon\left(\partial_{x} u_{\varepsilon, \alpha}\right)^{2}+\varepsilon \alpha \partial_{x} u_{\varepsilon, \alpha} \partial_{x x x}^{3} u_{\varepsilon, \alpha},
$$

where $\Gamma_{\varepsilon, \alpha}$ is defined in (5.2) and

$$
\Delta_{\varepsilon, \alpha}:=\alpha \partial_{t x}^{2} u_{\varepsilon, \alpha}+\frac{\alpha}{2}\left(\partial_{x} u_{\varepsilon, \alpha}\right)^{2}+\alpha u_{\varepsilon, \alpha} \partial_{x x}^{2} u_{\varepsilon, \alpha} .
$$

Arguing as in the proof of (5.5), using also (2.13)

$$
\left\|\partial_{x} u_{\varepsilon, \alpha} \Delta_{\varepsilon, \alpha}\right\|_{L^{1}\left(\mathbb{R}_{+} \times \mathbb{R}\right)} \leq \frac{C_{0}}{\sqrt{2}}\left(\frac{\sqrt{\alpha}}{\varepsilon} K_{0}+\frac{\sqrt{c_{1}} C_{0}^{2}}{2 \sqrt{2}} \frac{\sqrt{\alpha}}{\varepsilon}+\frac{\sqrt{\alpha}}{\varepsilon} K_{0}\right)=O\left(\frac{\sqrt{\alpha}}{\varepsilon}\right),
$$

and hence

$$
\partial_{x} u_{\varepsilon, \alpha} \Delta_{\varepsilon, \alpha} \rightarrow 0 \quad L^{1}\left(\mathbb{R}_{+} \times \mathbb{R}\right) \text { as } \varepsilon, \alpha \rightarrow 0 .
$$

Let $\varphi \in C_{c}^{\infty}\left(\mathbb{R}_{+} \times \mathbb{R}\right)$ be a nonnegative function. Using (4.2), (4.3), (5.4) and (5.7), we then calculate as follows:

$$
\begin{aligned}
& \int_{\mathbb{R}_{+} \times \mathbb{R}}\left[\partial_{t}\left(\frac{u_{\varepsilon, \alpha}^{2}}{2}\right)+\partial_{x} q\left(u_{\varepsilon, \alpha}\right)\right] \varphi d t d x \\
& =\int_{\mathbb{R}_{+} \times \mathbb{R}}\left[\partial_{x}\left(u_{\varepsilon, \alpha} \Gamma_{\varepsilon, \alpha}\right)-\partial_{x} u_{\varepsilon, \alpha} \Delta_{\varepsilon, \alpha}\right. \\
& \left.-\varepsilon\left(\partial_{x} u_{\varepsilon, \alpha}\right)^{2}+\varepsilon \alpha \partial_{x} u_{\varepsilon, \alpha} \partial_{x x x}^{3} u_{\varepsilon, \alpha}\right] \varphi d t d x \\
& \leq \int_{\mathbb{R}_{+} \times \mathbb{R}}\left[-u_{\varepsilon, \alpha} \Gamma_{\varepsilon, \alpha} \partial_{x} \varphi-\partial_{x} u_{\varepsilon, \alpha} \Delta_{\varepsilon, \alpha} \varphi\right. \\
& \left.\quad-\varepsilon \alpha\left(\partial_{x x}^{2} u_{\varepsilon, \alpha}\right)^{2} \varphi-\varepsilon \alpha \partial_{x} u_{\varepsilon, \alpha} \partial_{x x}^{2} u_{\varepsilon, \alpha} \partial_{x} \varphi\right] d t d x \\
& \quad+\left\|u_{\varepsilon, \alpha}\right\|_{L^{\infty}\left(\mathbb{R}_{+} ; L^{2}(\mathbb{R})\right)}\left\|\Gamma_{\varepsilon, \alpha}\right\|_{L^{2}\left(\mathbb{R}_{+} \times \mathbb{R}\right)}\left\|\partial_{x} \varphi\right\|_{L^{\infty}\left(\mathbb{R}_{+} \times \mathbb{R}\right)} \\
& \quad+\frac{\varepsilon \alpha}{2} \int_{\mathbb{R}_{+} \times \mathbb{R}}\left(\partial_{x} u_{\varepsilon, \alpha}\right)^{2} \partial_{x x}^{2} \varphi d t d x \\
& \leq C_{0}\left\|\Gamma_{\varepsilon, \alpha}\right\|_{L^{2}\left(\mathbb{R}_{+} \times \mathbb{R}\right)}\left\|\partial_{x} \varphi\right\|_{L^{\infty}}+\left\|\partial_{x} u_{\varepsilon, \alpha} \Delta_{\varepsilon, \alpha}\right\|_{L^{1}\left(\mathbb{R}_{+} \times \mathbb{R}\right)}\|\varphi\|_{L^{\infty}\left(\mathbb{R}_{+} \times \mathbb{R}\right)} \\
& \quad+\frac{\varepsilon \alpha}{2}\left\|\partial_{x} u_{\varepsilon, \alpha}\right\|_{L^{2}\left(\mathbb{R}_{+} \times \mathbb{R}\right)}^{2}\left\|\partial_{x x}^{2} \varphi\right\|_{L^{\infty}\left(\mathbb{R}_{+} \times \mathbb{R}\right)} \\
& \leq C_{0}\left\|\Gamma_{\varepsilon, \alpha}\right\|_{L^{2}\left(\mathbb{R}_{+} \times \mathbb{R}\right)}\left\|\partial_{x} \varphi\right\|_{L^{\infty}\left(\mathbb{R}_{+} \times \mathbb{R}\right)}+\left\|\partial_{x} u_{\varepsilon, \alpha} \Delta_{\varepsilon, \alpha}\right\|_{L^{1}}\|\varphi\|_{L^{\infty}\left(\mathbb{R}_{+} \times \mathbb{R}\right)} \\
& \quad+\frac{C_{0}^{2} \alpha}{4}\left\|\partial_{x x}^{2} \varphi\right\|_{L^{\infty}\left(\mathbb{R}_{+} \times \mathbb{R}\right)} \rightarrow 0,
\end{aligned}
$$


which proves the lemma.

Proof of Theorem 2.1. Assertions $(i),(i i)$, and $(i i i)$ are contained in Lemma 5.2, while Assertion (iv) follows from Lemma 5.3.

\section{REFERENCES}

[1] R. Beals, D. H. Sattinger, and J. Szmigielski. Acoustic scattering and the extended Korteweg-de Vries hierarchy. Adv. Math., 140(2):190-206, 1998.

[2] T. B. Benjamin, J. L. Bona, and J. J. Mahony. Model equations for long waves in nonlinear dispersive systems. Philos. Trans. Roy. Soc. London Ser. A, 272(1220):47-78, 1972.

[3] R. Camassa and D. D. Holm. An integrable shallow water equation with peaked solitons. Phys. Rev. Lett., 71(11):1661-1664, 1993.

[4] B. Cassis. Compensated compactness applied to a singular perturbed fourth order p.d.e. Comm. Partial Differential Equations, 14(5):619-634, 1989.

[5] G. M. Coclite, H. Holden, and K. H. Karlsen. Global weak solutions to a generalized hyperelastic-rod wave equation. E-print, Department of Mathematics, University of Oslo, 2004.

[6] G. M. Coclite, H. Holden, and K. H. Karlsen. Wellposedness for a parabolic-elliptic system. to appear on Discrete Contin. Dynam. Systems.

[7] A. Constantin. Existence of permanent and breaking waves for a shallow water equation: a geometric approach. Ann. Inst. Fourier (Grenoble), 50(2):321-362, 2000.

[8] A. Constantin. On the scattering problem for the Camassa-Holm equation. R. Soc. Lond. Proc. Ser. A Math. Phys. Eng. Sci., 457(2008):953-970, 2001.

[9] A. Constantin and J. Escher. Global existence and blow-up for a shallow water equation. Ann. Scuola Norm. Sup. Pisa Cl. Sci. (4), 26(2):303-328, 1998.

[10] A. Constantin and J. Escher. Global weak solutions for a shallow water equation. Indiana Univ. Math. J., 47(4):1527-1545, 1998.

[11] A. Constantin and J. Escher. Wave breaking for nonlinear nonlocal shallow water equations. Acta Math., 181(2):229-243, 1998.

[12] A. Constantin and H. P. McKean. A shallow water equation on the circle. Comm. Pure Appl. Math., 52(8):949982, 1999.

[13] A. Constantin and L. Molinet. Global weak solutions for a shallow water equation. Comm. Math. Phys., 211(1):45-61, 2000.

[14] J. M. C. Correia and P. G. Lefloch. Nonlinear diffusive-dispersive limits for multidimensional conservation laws. In Advances in nonlinear partial differential equations and related areas (Beijing, 1997), pages 103-123. World Sci. Publishing, River Edge, NJ, 1998.

[15] H.-H. Dai and Y. Huo. Solitary shock waves and other travelling waves in a general compressible hyperelastic rod. R. Soc. Lond. Proc. Ser. A Math. Phys. Eng. Sci., 456(1994):331-363, 2000.

[16] R. Danchin. A few remarks on the Camassa-Holm equation. Differential Integral Equations, 14(8):953-988, 2001.

[17] R. Danchin. A note on well-posedness for Camassa-Holm equation. J. Differential Equations, 192(2):429-444, 2003.

[18] C. De Lellis, F. Otto, and M. Westdickenberg. Minimal entropy condition for burgers equation. Quart. Appl. Math. To appear.

[19] B. Fuchssteiner and A. S. Fokas. Symplectic structures, their Bäcklund transformations and hereditary symmetries. Phys. D, 4(1):47-66, 1981/82.

[20] A. A. Himonas and G. Misiołek. The Cauchy problem for an integrable shallow-water equation. Differential Integral Equations, 14(7):821-831, 2001.

[21] H. Holden and N. H. Risebro. Front tracking for hyperbolic conservation laws, volume 152 of Applied Mathematical Sciences. Springer-Verlag, New York, 2002.

[22] D. D. Holm and M. F. Staley. Wave structure and nonlinear balances in a family of evolutionary PDEs. SIAM J. Appl. Dyn. Syst., 2(3):323-380 (electronic), 2003.

[23] S. Hwang. Kinetic decomposition for singularly perturbed higher order partial differential equations. J. Differential Equations, 200(2):191-205, 2004.

[24] S. Hwang and A. E. Tzavaras. Kinetic decomposition of approximate solutions to conservation laws: application to relaxation and diffusion-dispersion approximations. Comm. Partial Differential Equations, 27(5-6):1229$1254,2002$.

[25] R. S. Johnson. Camassa-Holm, Korteweg-de Vries and related models for water waves. J. Fluid Mech., 455:63$82,2002$.

[26] C. I. Kondo and P. G. Lefloch. Zero diffusion-dispersion limits for scalar conservation laws. SIAM J. Math. Anal., 33(6):1320-1329 (electronic), 2002.

[27] S. N. Kružkov. First order quasi-linear equations in several independent variables. Math. USSR Sbornik, 10(2):217-243, 1970. 
[28] P. D. Lax and C. D. Levermore. The small dispersion limit of the Korteweg-de Vries equation. I. Comm. Pure Appl. Math., 36(3):253-290, 1983.

[29] P. D. Lax and C. D. Levermore. The small dispersion limit of the Korteweg-de Vries equation. II. Comm. Pure Appl. Math., 36(5):571-593, 1983.

[30] P. D. Lax and C. D. Levermore. The small dispersion limit of the Korteweg-de Vries equation. III. Comm. Pure Appl. Math., 36(6):809-829, 1983.

[31] P. G. LeFloch. Hyperbolic systems of conservation laws. Lectures in Mathematics ETH Zürich. Birkhäuser Verlag, Basel, 2002. The theory of classical and nonclassical shock waves.

[32] P. G. LeFloch and R. Natalini. Conservation laws with vanishing nonlinear diffusion and dispersion. Nonlinear Anal., 36(2, Ser. A: Theory Methods):213-230, 1999.

[33] Y. A. Li and P. J. Olver. Well-posedness and blow-up solutions for an integrable nonlinearly dispersive model wave equation. J. Differential Equations, 162(1):27-63, 2000.

[34] Y. G. Lu. Convergence of solutions to nonlinear dispersive equations without convexity conditions. Appl. Anal., 31(4):239-246, 1989.

[35] B. J. Lucier. On Sobolev regularizations of hyperbolic conservation laws. Comm. Partial Differential Equations, 10(1):1-28, 1985.

[36] F. Murat. L'injection du cône positif de $H^{-1}$ dans $W^{-1, q}$ est compacte pour tout $q<2$. J. Math. Pures Appl. (9), 60(3):309-322, 1981.

[37] E. Y. Panov. Uniqueness of the solution of the Cauchy problem for a first-order quasilinear equation with an admissible strictly convex entropy. Mat. Zametki, 55(5):116-129, 159, 1994.

[38] G. Rodríguez-Blanco. On the Cauchy problem for the Camassa-Holm equation. Nonlinear Anal., 46(3, Ser. A: Theory Methods):309-327, 2001.

[39] M. E. Schonbek. Convergence of solutions to nonlinear dispersive equations. Comm. Partial Differential Equations, 7(8):959-1000, 1982.

[40] A. Szepessy. An existence result for scalar conservation laws using measure valued solutions. Comm. Partial Differential Equations, 14(10):1329-1350, 1989.

[41] E. Tadmor. Burgers' equation with vanishing hyper-viscosity. Commun. Math. Sci., 2(2):317-324, 2004.

[42] L. Tartar. Compensated compactness and applications to partial differential equations. In Nonlinear analysis and mechanics: Heriot-Watt Symposium, Vol. IV, pages 136-212. Pitman, Boston, Mass., 1979.

[43] Z. Xin and P. Zhang. On the weak solutions to a shallow water equation. Comm. Pure Appl. Math., 53(11):1411$1433,2000$.

[44] Z. Xin and P. Zhang. On the uniqueness and large time behavior of the weak solutions to a shallow water equation. Comm. Partial Differential Equations, 27(9-10):1815-1844, 2002.

[45] H. Zhao. A note on the Cauchy problem to a class of nonlinear dispersive equations with singular initial data. Nonlinear Anal., 42(2, Ser. A: Theory Methods):251-270, 2000.

(Giuseppe Maria Coclite)

Centre of Mathematics for Applications (CMA)

UNIVERSITY OF OSLO

P.O. Box 1053, BLINDERN

N-0316 Oslo, Norway

E-mail address: giuseppec@math.uio.no

(Kenneth Hvistendahl Karlsen)

Centre of Mathematics for Applications (CMA)

UNIVERSITY OF OSLO

P.O. Box 1053, BLINDERN

N-0316 OSLO, NorWAY

E-mail address: kennethk@math.uio.no

URL: http://www.math.uio.no/ kennethk/ 